\title{
Vanishing of cohomology and parameter rigidity of actions of solvable Lie groups, II
}

\author{
HIROKAZU MARUHASHI \\ The University of Tokyo, 3-8-1 Komaba, Meguro, Tokyo, 153-8914, Japan \\ (e-mail:maruhashihirokazu@gmail.com)
}

(Received 3 January 2019 and accepted in revised form 21 January 2020)

Abstract. Let $M \stackrel{\rho_{0}}{\curvearrowleft} S$ be a $C^{\infty}$ locally free action of a connected simply connected solvable Lie group $S$ on a closed manifold $M$. Roughly speaking, $\rho_{0}$ is parameter rigid if any $C^{\infty}$ locally free action of $S$ on $M$ having the same orbits as $\rho_{0}$ is $C^{\infty}$ conjugate to $\rho_{0}$. In this paper we prove two types of result on parameter rigidity.

First let $G$ be a connected semisimple Lie group with finite center of real rank at least 2 without compact factors nor simple factors locally isomorphic to $\mathrm{SO}_{0}(n, 1)(n \geq 2)$ or $\mathrm{SU}(n, 1)(n \geq 2)$, and let $\Gamma$ be an irreducible cocompact lattice in $G$. Let $G=K A N$ be an Iwasawa decomposition. We prove that the action $\Gamma \backslash G \curvearrowleft A N$ by right multiplication is parameter rigid. One of the three main ingredients of the proof is the rigidity theorems of Pansu, and Kleiner and Leeb on the quasi-isometries of Riemannian symmetric spaces of non-compact type.

Secondly we show that if $M \stackrel{\rho_{0}}{n} S$ is parameter rigid, then the zeroth and first cohomology of the orbit foliation of $\rho_{0}$ with certain coefficients must vanish. This is a partial converse to the results in the author's [Vanishing of cohomology and parameter rigidity of actions of solvable Lie groups. Geom. Topol. 21(1) (2017), 157-191], where we saw sufficient conditions for parameter rigidity in terms of vanishing of the first cohomology with various coefficients.

Key words: parameter rigidity, leafwise cohomology, quasi-isometries

2020 Mathematics Subject Classification: 17B56, 37C85, 37C86 (Primary); 22E25, 22E40, 51F30 (Secondary)

\section{Contents}

1 Introduction

2 Parameter rigidity of the action of $A N$ on $\Gamma \backslash G$

3 Preliminaries 
$\begin{array}{ll}3.2 & \text { A sufficient condition for parameter rigidity } \\ 3.3027\end{array}$

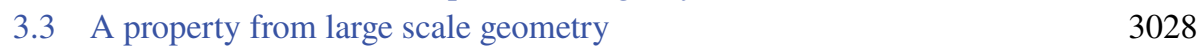

4 Reduction of the proof of Theorem 2 to Proposition $12 \quad 3029$

5 Proof of Proposition $12 \quad 3032$

5.1 The case where $G$ is of real rank at least 2 or is locally isomorphic to $\operatorname{Sp}(n, 1)(n \geq 2)$ or $F_{4}^{-20}$

5.2 The case where $G$ is of real rank $1 \quad 3041$

6 Necessary conditions for parameter rigidity 3047

7 Vanishing of $H^{0}$ - proof of Theorem $28 \quad 3049$

8 Vanishing of $H^{1}$-proof of Theorem $32 \quad 3055$

$\begin{array}{lr}\text { Acknowledgements } & 3059\end{array}$

References $\quad 3059$

\section{Introduction}

This paper consists of two parts. The first part, $\S \S 2-5$, deals with parameter rigidity of certain actions. Section 2 serves as the introduction for the first part. The second part, $\S \S 6-8$, is on necessary conditions for parameter rigidity in terms of vanishing of cohomology. Section 6 serves as the introduction for the second part.

\section{Parameter rigidity of the action of $A N$ on $\Gamma \backslash G$}

Let $M \stackrel{\rho_{0}}{\curvearrowleft} S$ be a $C^{\infty}$ locally free (i.e. the isotropy subgroup of every point is discrete) action of a connected simply connected solvable Lie group $S$ on a closed $C^{\infty}$ manifold $M$. Let $\mathcal{F}$ be the set of all orbits of $\rho_{0}$, which is called the orbit foliation of $\rho_{0}$ and actually is a $C^{\infty}$ foliation of $M$. We say $\rho_{0}$ is parameter rigid if every $C^{\infty}$ locally free action $M \stackrel{\rho}{\curvearrowleft} S$ with the same orbit foliation as that of $\rho_{0}$ is parameter equivalent to $\rho_{0}$. (We do not assume that $\rho$ is close to $\rho_{0}$ in some topology.) Here parameter equivalence between $\rho$ and $\rho_{0}$ means the following. There exist a diffeomorphism $F$ of $M$ and an automorphism $\Phi$ of $S$ such that:

- $\quad F\left(\rho_{0}(x, s)\right)=\rho(F(x), \Phi(s))$ for all $x \in M$ and $s \in S$;

- $\quad$ the map $F$ preserves each leaf of $\mathcal{F}$; that is, $F(L) \subset L$ for all $L \in \mathcal{F}$;

- the map $F$ is $C^{0}$ homotopic to the identity map of $M$ through $C^{\infty}$ maps which preserve each leaf of $\mathcal{F}$.

For example a linear flow on a torus is parameter rigid if and only if the velocity vector satisfies the Diophantus condition.

In [11] and [12] Katok and Spatzier proved the following.

THEOREM 1. (Katok-Spatzier) Let $G$ be a connected semisimple Lie group with finite center of real rank at least 2 without compact factors nor simple factors locally isomorphic to $\mathrm{SO}_{0}(n, 1)(n \geq 2)$ or $\mathrm{SU}(n, 1)(n \geq 2)$, and let $\Gamma$ be an irreducible cocompact lattice in $G$. Let $G=K A N$ be an Iwasawa decomposition. Then the action $\Gamma \backslash G \curvearrowleft A$ by right multiplication is parameter rigid.

This is proved using representation theory of semisimple Lie groups and has led to considerable subsequent research. In this paper we prove the following, based on the above theorem and applying large scale geometry. 
THEOREM 2. Under the same assumptions as Theorem 1, the action $\Gamma \backslash G \curvearrowleft A N$ by right multiplication is parameter rigid.

We give a proof of this theorem in $\S 4$ and $\S 5$ after recalling the results in Maruhashi [17] in $\S 3$. The proof is a combination of the following three steps.

(1) Vanishing of cohomology $\Rightarrow$ parameter rigidity. This is the sufficient condition for parameter rigidity proved in [17]. In the current article this is Theorem 4.

(2) Cohomology vanishing results. These are by Katok and Spatzier [11, 12] and Kanai [10]. See Theorem 10 and Corollary 11 in this paper.

(3) Bridging the gap between Step 1 and Step 2. This is because the cohomology vanishing results are available only for finitely many coefficients, while the sufficient condition for parameter rigidity requires vanishing of cohomology for seemingly many more coefficients. Here we use Proposition 6, which shows the relevance to large scale geometry. Then the main point is that our acting group $A N$ is isometric to $G / K$ by the Iwasawa decomposition $G=A N K$. So we can use the rigidity theorems of Pansu [18] and Kleiner and Leeb [13] on quasi-isometries of symmetric spaces, and a certain rigidity property of quasi-isometries of hyperbolic spaces proved in Farb and Mosher [5] and Reiter Ahlin [19].

Theorem 2 shows a contrast between the higher-rank case and $\widetilde{\operatorname{PSL}}(2, \mathbb{R})$, the universal cover of $\operatorname{PSL}(2, \mathbb{R})$, for which Asaoka [1] gives (generally) non-trivial orbit-preserving deformations of the actions of $A N$ by right multiplication.

THEOREM 3. (Asaoka [1]) Let $\Gamma$ be a cocompact lattice in $\widetilde{\mathrm{PSL}}(2, \mathbb{R})$ and let

$$
A=\left\{\left(\begin{array}{ll}
a & \\
& a^{-1}
\end{array}\right) \mid a>0\right\}, \quad N=\left\{\left(\begin{array}{ll}
1 & b \\
& 1
\end{array}\right) \mid b \in \mathbb{R}\right\} .
$$

Let $\Phi_{\Gamma}$ be the flow on $\Gamma \backslash \widetilde{\mathrm{PSL}}(2, \mathbb{R})$ defined by the action of $A$ by right multiplication, $\mathcal{P}$ the set of oriented periodic orbits of $\Phi_{\Gamma}$, and $\tau(\gamma)$ the period of $\gamma$ for $\gamma \in \mathcal{P}$. Consider

$$
\Delta_{\Gamma}=\left\{a \in H^{1}(\Gamma \backslash \widetilde{\operatorname{PSL}}(2, \mathbb{R}) ; \mathbb{R}) \mid \sup _{\gamma \in \mathcal{P}} \frac{|a(\gamma)|}{\tau(\gamma)}<1\right\},
$$

which is an open neighborhood of 0 in $H^{1}(\Gamma \backslash \widetilde{\operatorname{PSL}}(2, \mathbb{R}) ; \mathbb{R})$. Then there exists an analytic locally free action $\rho_{a}$ of $A N$ on $\Gamma \backslash \widetilde{\mathrm{PSL}}(2, \mathbb{R})$ for each $a \in \Delta_{\Gamma}$ with the following properties.

- The action $\rho_{0}$ is defined by right multiplication.

- All the actions $\rho_{a}$ have the same orbit foliation $\mathcal{F}$.

- The actions $\rho_{a}$ and $\rho_{a^{\prime}}$ are not parameter equivalent if $a \neq a^{\prime}$.

- Any $C^{\infty}$ locally free action of $A N$ whose orbit foliation is $\mathcal{F}$ is parameter equivalent to $\rho_{a}$ for some $a \in \Delta_{\Gamma}$.

- $\quad$ The action $\rho_{a}$ does not preserve any $C^{0}$ volume form on $\Gamma \backslash \widetilde{\mathrm{PSL}}(2, \mathbb{R})$ except when $a=0$.

We also know how the action $\rho_{a}$ is controlled by the cohomology class $a$, but we refer the reader to [1] for that and more information. Note that the above deformation is different from the non-orbit-preserving deformation coming from the deformation of the lattice, 
whose deformation space has the dimension equal to that of Teichmüller space, because such deformations are necessarily $C^{0}$ volume-preserving.

\section{Preliminaries}

This section is a summary of the results we need later, proved in Maruhashi [17]. See [17] for the details. In this paper Lie algebras are denoted by the corresponding lowercase Fraktur of the corresponding Lie groups. The symbol $\Gamma(\cdot)$ denotes the set of all $C^{\infty}$ sections of a vector bundle.

3.1. Leafwise cohomology. Let $M \stackrel{\rho_{0}}{\curvearrowleft} S$ be a $C^{\infty}$ locally free action of a connected simply connected solvable Lie group $S$ on a closed manifold $M$ with the orbit foliation $\mathcal{F}$. Let $\omega_{0} \in \Gamma(\operatorname{Hom}(T \mathcal{F}, \mathfrak{s}))$ denote the canonical 1 -form of $\rho_{0}$; i.e. $\left(\omega_{0}\right)_{x}: T_{x} \mathcal{F} \rightarrow \mathfrak{s}$ for $x \in M$ is defined as the inverse of the derivative at the identity of the map $S \rightarrow M$, $s \mapsto \rho_{0}(x, s)$. Let

$$
d_{\mathcal{F}}: \Gamma\left(\bigwedge^{p} T^{*} \mathcal{F}\right) \rightarrow \Gamma\left(\bigwedge^{p+1} T^{*} \mathcal{F}\right)
$$

be the leafwise exterior derivative of $\mathcal{F}$, defined by the same formula as the usual exterior derivative. Then $\omega_{0}$ satisfies the Maurer-Cartan equation $d_{\mathcal{F}} \omega_{0}+\left[\omega_{0}, \omega_{0}\right]=0$. Here $d_{\mathcal{F}} \omega_{0}$ and $\left[\omega_{0}, \omega_{0}\right]$ are defined by

$$
d_{\mathcal{F}} \omega_{0}(X, Y)=X \omega_{0}(Y)-Y \omega_{0}(X)-\omega_{0}([X, Y])
$$

and

$$
\left[\omega_{0}, \omega_{0}\right](X, Y)=\left[\omega_{0}(X), \omega_{0}(Y)\right]
$$

for $X, Y \in \Gamma(T \mathcal{F})$. Let $\mathfrak{s} \stackrel{\pi}{\curvearrowright} V$ be a representation of $\mathfrak{s}$ on a finite-dimensional real vector space $V$. Then $\pi \omega_{0} \in \Gamma(\operatorname{Hom}(T \mathcal{F}, \operatorname{End}(V)))$ satisfies

$$
d_{\mathcal{F}} \pi \omega_{0}+\left[\pi \omega_{0}, \pi \omega_{0}\right]=0 .
$$

We regard $\pi \omega_{0}$ as the connection form of a flat $\mathcal{F}$-partial connection $\nabla$ of the trivial vector bundle $M \times V \rightarrow M$ relative to any global frame of the bundle which has constant $V$ components; i.e. $\nabla_{X} v=\pi\left(\omega_{0}(X)\right) v$ for $X \in \Gamma(T \mathcal{F})$ and $v \in V$, where $v$ is regarded as a section of $M \times V \rightarrow M$. Hence $\nabla \xi=d_{\mathcal{F}} \xi+\pi \omega_{0} \xi$ for general $\xi \in \Gamma(V)$. The exterior derivative of $\nabla$ is

$$
\begin{aligned}
\Gamma\left(\bigwedge^{p} T^{*} \mathcal{F} \otimes V\right) & \rightarrow \Gamma\left(\bigwedge^{p+1} T^{*} \mathcal{F} \otimes V\right) \\
\omega & \mapsto d_{\mathcal{F}} \omega+\pi \omega_{0} \wedge \omega,
\end{aligned}
$$

where our definition of exterior product is

$$
\left(\pi \omega_{0} \wedge \omega\right)\left(X_{1}, \ldots, X_{p+1}\right)=\sum_{i=1}^{p+1}(-1)^{i+1} \pi \omega_{0}\left(X_{i}\right) \omega\left(X_{1}, \ldots, \widehat{X_{i}}, \ldots, X_{p+1}\right) .
$$


The square of this operator is zero by the flatness. The cohomology $H^{*}(\mathcal{F} ; \mathfrak{s} \stackrel{\pi}{\curvearrowright} V)$ of this complex is the leafwise cohomology of $\mathcal{F}$ with coefficient $\pi$. Recall that the cohomology $H^{*}(\mathfrak{s} ; \mathfrak{s} \stackrel{\pi}{\curvearrowright} V)$ of the Lie algebra $\mathfrak{s}$ with coefficient $\pi$ is obtained from the complex $\operatorname{Hom}\left(\bigwedge^{*} \mathfrak{s}, V\right)$. We have an injective cochain map

$$
\begin{aligned}
\operatorname{Hom}\left(\bigwedge^{*} \mathfrak{s}, V\right) & \hookrightarrow \Gamma\left(\bigwedge^{*} T^{*} \mathcal{F} \otimes V\right) \\
\varphi & \mapsto \omega_{0}^{*} \varphi,
\end{aligned}
$$

where $\omega_{0}^{*}$ is the pullback by $\omega_{0}$. Then by [17, Lemma 2.1.3] the induced map

$$
H^{*}(\mathfrak{s} ; \mathfrak{s} \stackrel{\pi}{\curvearrowright} V) \rightarrow H^{*}(\mathcal{F} ; \mathfrak{s} \stackrel{\pi}{\curvearrowright} V)
$$

is injective and we see $H^{*}(\mathfrak{s} ; \mathfrak{s} \stackrel{\pi}{\curvearrowright} V)$ as a subspace of $H^{*}(\mathcal{F} ; \mathfrak{s} \stackrel{\pi}{\curvearrowright} V)$.

3.2. A sufficient condition for parameter rigidity. Let $\mathfrak{n}$ denote the nilradical of $\mathfrak{s}$. We have $[\mathfrak{s}, \mathfrak{s}] \subset \mathfrak{n}$. Take a subspace $\mathfrak{h}$ such that $[\mathfrak{s}, \mathfrak{s}] \subset \mathfrak{h} \subset \mathfrak{n}$. Then $\mathfrak{h}$ is a nilpotent ideal of $\mathfrak{s}$. Let

$$
\mathfrak{h} \supset \mathfrak{h}^{2} \supset \cdots \supset \mathfrak{h}^{d} \supset 0
$$

be the lower central series of $\mathfrak{h}$. This filtration of $\mathfrak{h}$ is invariant with respect to $\mathfrak{s} \stackrel{\text { ad }}{\curvearrowright} \mathfrak{h}$. Let

$$
\mathfrak{s} \stackrel{\text { ad }}{\curvearrowright} \operatorname{Gr}(\mathfrak{h})=\bigoplus_{i=1}^{d} \mathfrak{h}^{i} / \mathfrak{h}^{i+1}
$$

be the associated graded quotient. Since $\mathfrak{h}$ acts trivially, we get $\mathfrak{s} / \mathfrak{h} \stackrel{\text { ad }}{\curvearrowright} \operatorname{Gr}(\mathfrak{h})$.

Let $\mathcal{A}(\mathcal{F}, S)$ be the set of all $C^{\infty}$ locally free actions $M \curvearrowleft S$ with the orbit foliation $\mathcal{F}$. Let $\rho \in \mathcal{A}(\mathcal{F}, S)$, and let $\omega$ denote the canonical 1 -form of $\rho$. Let $p: \mathfrak{s} \rightarrow \mathfrak{s} / \mathfrak{h}$ denote the natural projection. Applying $p$ to $d_{\mathcal{F}} \omega+[\omega, \omega]=0$, we get $d_{\mathcal{F}} p \omega=0$. Assume $H^{1}(\mathcal{F})=H^{1}(\mathfrak{s})$. Then $[p \omega] \in H^{1}(\mathcal{F} ; \mathfrak{s} / \mathfrak{h})=H^{1}(\mathfrak{s} ; \mathfrak{s} / \mathfrak{h})$. So there exist a unique linear map $\varphi_{\rho}: \mathfrak{s} \rightarrow \mathfrak{s} / \mathfrak{h}$ which vanishes on $[\mathfrak{s}, \mathfrak{s}]$ and a $C^{\infty}$ map $h: M \rightarrow \mathfrak{s} / \mathfrak{h}$ such that

$$
p \omega=\varphi_{\rho} \omega_{0}+d_{\mathcal{F}} h .
$$

The map $\varphi_{\rho}$ is surjective by [17, Lemma 2.2.2].

THEOREM 4. (Maruhashi [17]) If

$$
H^{1}(\mathcal{F})=H^{1}(\mathfrak{s})
$$

and

$$
H^{1}\left(\mathcal{F} ; \mathfrak{s} \stackrel{\operatorname{ad} \circ \varphi_{\rho}}{\curvearrowright} \operatorname{Gr}(\mathfrak{h})\right)=H^{1}\left(\mathfrak{s} ; \mathfrak{s} \stackrel{\operatorname{ad} \circ \varphi_{\rho}}{\curvearrowright} \operatorname{Gr}(\mathfrak{h})\right)
$$

for some $\mathfrak{h}$ and for all $\rho \in \mathcal{A}(\mathcal{F}, S)$, then $M \stackrel{\rho_{0}}{\curvearrowleft} S$ is parameter rigid.

See [17, Theorem 2.2.5] for this theorem. 
3.3. A property from large scale geometry. Let $\rho \in \mathcal{A}(\mathcal{F}, S)$ and let $a_{\rho}: M \times S \rightarrow S$ be the unique $C^{\infty}$ map satisfying

$$
\rho_{0}(x, s)=\rho\left(x, a_{\rho}(x, s)\right) \quad \text { and } \quad a_{\rho}(x, 1)=1
$$

for all $x \in M$ and $s \in S$. The map $a_{\rho}$ is defined since $\rho_{0}$ and $\rho$ have the same orbit foliation. It is known that $a_{\rho}$ is a cocycle over $\rho_{0}$.

Let $X, B$ be metric spaces. A surjective map $p: X \rightarrow B$ is a distance-respecting projection if

$$
d\left(b, b^{\prime}\right)=d\left(p^{-1}(b), p^{-1}\left(b^{\prime}\right)\right)=d_{\mathcal{H}}\left(p^{-1}(b), p^{-1}\left(b^{\prime}\right)\right)
$$

holds for all $b, b^{\prime} \in B$, where

$$
d\left(p^{-1}(b), p^{-1}\left(b^{\prime}\right)\right)=\inf \left\{d\left(x, x^{\prime}\right) \mid x \in p^{-1}(b), x^{\prime} \in p^{-1}\left(b^{\prime}\right)\right\}
$$

and $d_{\mathcal{H}}$ denotes the Hausdorff distance. Let $p: X \rightarrow B$ and $p^{\prime}: X^{\prime} \rightarrow B^{\prime}$ be distancerespecting projections. A diagram

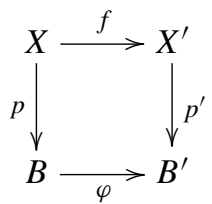

is fiber-respecting, or $f$ is fiber-respecting over $\varphi$, if $f$ and $\varphi$ are maps and there exists a constant $C>0$ such that $d_{\mathcal{H}}\left(f\left(p^{-1}(b)\right),\left(p^{\prime}\right)^{-1}(\varphi(b))\right)<C$ for all $b \in B$.

Proposition 5. Let $G$ be a connected Lie group and $H$ a connected normal closed subgroup of $G$. Take an inner product of $\mathfrak{g}$. Endow $\mathfrak{g} / \mathfrak{h}$ with the inner product for which the restriction $\mathfrak{h}^{\perp} \stackrel{\sim}{\rightarrow} \mathfrak{g} / \mathfrak{h}$ of the projection $\mathfrak{g} \rightarrow \mathfrak{g} / \mathfrak{h}$ is an isometry. Give $G$ and $G / H$ left-invariant Riemannian metrics corresponding to these inner products. Then the projection $p: G \rightarrow G / H$ is a distance-respecting projection.

Proof. This follows from [17, Lemma 4.1.1] by noting that $H \stackrel{\text { Ad }}{\curvearrowright} \mathfrak{g} / \mathfrak{h}$ is trivial.

Assume $H^{1}(\mathcal{F})=H^{1}(\mathfrak{s})$ for an action $M \stackrel{\rho_{0}}{\curvearrowleft} S$ and let $\rho \in \mathcal{A}(\mathcal{F}, S)$ and $\varphi_{\rho}: \mathfrak{s} \rightarrow \mathfrak{s} / \mathfrak{h}$, $a_{\rho}: M \times S \rightarrow S$ as above. Let $K_{\rho}$ and $H$ be the Lie subgroups corresponding to $\operatorname{ker} \varphi_{\rho}$ and $\mathfrak{h}$. Then $S / K_{\rho}$ and $S / H$ are vector groups. Let $\tilde{\varphi}_{\rho}: S / K_{\rho} \rightarrow S / H$ be the linear isomorphism with differential $\varphi_{\rho}: \mathfrak{s} / \operatorname{ker} \varphi_{\rho} \simeq \mathfrak{s} / \mathfrak{h}$.

Proposition 6. (Maruhashi [17]) For any $\rho \in \mathcal{A}(\mathcal{F}, S), x \in M$ and $\mathfrak{h}$, consider the diagram

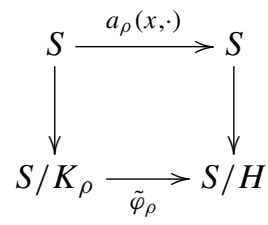


where the vertical maps are the natural projections. Fix an inner product of $\mathfrak{s}$ and give $S$, $S / K_{\rho}$ and $S / H$ the left-invariant Riemannian metrics considered in Proposition 5. Then $a_{\rho}(x, \cdot)$ is a fiber-respecting bi-Lipschitz diffeomorphism over $\tilde{\varphi}_{\rho} .\left(\right.$ In particular $a_{\rho}(x, \cdot)$ is a quasi-isometry.)

See [17, Proposition 4.1.4] for this proposition.

\section{Reduction of the proof of Theorem 2 to Proposition 12}

Let $G$ be a connected semisimple Lie group. Fix a Cartan decomposition $\mathfrak{g}=\mathfrak{k} \oplus \mathfrak{p}$ and a maximal abelian subspace $\mathfrak{a}$ of $\mathfrak{p}$. Let $\Sigma$ be the restricted root system of $(\mathfrak{g}, \mathfrak{a})$ and fix a positive system $\Sigma_{+}$of $\Sigma$. Let $\mathfrak{n}=\bigoplus_{\lambda \in \Sigma_{+}} \mathfrak{g}_{\lambda}$, where

$$
\mathfrak{g}_{\lambda}=\{X \in \mathfrak{g} \mid[H, X]=\lambda(H) X \text { for all } H \in \mathfrak{a}\}
$$

is a restricted root space. Let $K, A$ and $N$ be the Lie subgroups corresponding to $\mathfrak{k}, \mathfrak{a}$ and $\mathfrak{n}$. Then $G=K A N$ is an Iwasawa decomposition. The group $A N$ is a connected simply connected solvable Lie group and its Lie algebra is $\mathfrak{a n}=\mathfrak{n} \rtimes \mathfrak{a}$.

It is easy to show that $\mathfrak{n}$ is the nilradical of $\mathfrak{a n}$ and $\mathfrak{n}=[\mathfrak{a n}, \mathfrak{a n}]$. So we must take $\mathfrak{h}=\mathfrak{n}$ to apply Theorem 4 . Then

$$
\mathfrak{a n} / \mathfrak{n}=\mathfrak{a} \stackrel{\mathrm{ad}}{\curvearrowright} \operatorname{Gr}(\mathfrak{n})=\bigoplus_{i \geq 1} \mathfrak{n}^{i} / \mathfrak{n}^{i+1}
$$

is isomorphic to

$$
\mathfrak{a} \stackrel{\mathrm{ad}}{\curvearrowright} \mathfrak{n}=\bigoplus_{\lambda \in \Sigma_{+}} \mathfrak{g}_{\lambda}
$$

To apply Theorem 4, we must show $H^{1}(\mathcal{F})=H^{1}(\mathfrak{a n})$ and then calculate cohomology with coefficient

$$
\mathfrak{a n} \stackrel{\operatorname{ad} \circ \varphi_{\rho}}{\curvearrowright} \operatorname{Gr}(\mathfrak{n}), \quad \text { i.e. } \quad \mathfrak{a n} \stackrel{\operatorname{ad} \circ \varphi_{\rho}}{\curvearrowright} \mathfrak{n}=\bigoplus_{\lambda \in \Sigma_{+}} \mathfrak{g}_{\lambda},
$$

for any $\rho \in \mathcal{A}(\mathcal{F}, A N)$, where $\varphi_{\rho}: \mathfrak{a n} \rightarrow \mathfrak{a}$. Note that $\operatorname{ker} \varphi_{\rho}=\mathfrak{n}$ and $\left.\varphi_{\rho}\right|_{\mathfrak{a}} \in \operatorname{GL}(\mathfrak{a})$. The $\mathfrak{g}_{\lambda}$-component $\mathfrak{a n} \stackrel{\operatorname{ad} \circ \varphi_{\rho}}{\curvearrowright} \mathfrak{g}_{\lambda}$ in (1) is a direct sum of the one-dimensional representation $\mathfrak{a n} \stackrel{\lambda \circ \varphi_{\rho}}{\curvearrowright} \mathbb{R}$. Therefore, we get the following.

Lemma 7. Let $G$ be a connected semisimple Lie group. Fix a Cartan decomposition $\mathfrak{g}=$ $\mathfrak{k} \oplus \mathfrak{p}$, a maximal abelian subspace $\mathfrak{a}$ of $\mathfrak{p}$ with the associated restricted root system $\Sigma$, and a positive system $\Sigma_{+}$of $\Sigma$. Let $\mathcal{F}$ be the orbit foliation of a $C^{\infty}$ locally free action $M \stackrel{\rho_{0}}{\curvearrowleft} A N$ on a closed manifold $M$. If

$$
H^{1}(\mathcal{F})=H^{1}(\mathfrak{a n})
$$

and

$$
H^{1}\left(\mathcal{F} ; \mathfrak{a n} \stackrel{\lambda \circ \varphi_{\rho}}{\curvearrowright} \mathbb{R}\right)=H^{1}\left(\mathfrak{a n} ; \mathfrak{a n} \stackrel{\lambda \circ \varphi_{\rho}}{\curvearrowright} \mathbb{R}\right)
$$

for any $\lambda \in \Sigma_{+}$and $\rho \in \mathcal{A}(\mathcal{F}, A N)$, then $\rho_{0}$ is parameter rigid. 
Before proving Theorem 2 we remark that the same result but with a stronger assumption of real rank at least 3 follows easily from the following result of Kononenko [15, Theorem 8.2].

THEOREM 8. (Kononenko [15]) Let $G$ be a connected semisimple Lie group with finite center of real rank at least 3 whose simple factors are of real rank at least 2, and let $\Gamma$ be an irreducible cocompact lattice in $G$. Let $G=K A N$ be an Iwasawa decomposition. Take $\mu: \mathfrak{a n} \rightarrow \mathbb{R}$ to be any non-zero linear function which vanishes on $\mathfrak{n}$, and let $\tilde{\mu}: A N \rightarrow$ $\operatorname{GL}(1, \mathbb{R})$ be the homomorphism with differential $\mu$. Then any $\tilde{\mu}$-twisted $C^{\infty}$ cocycle over the action $\Gamma \backslash G \curvearrowleft A N$ by right multiplication is $C^{\infty}$ cohomologous to a constant cocycle. Equivalently we have

$$
H^{1}(\mathcal{F} ; \mathfrak{a n} \stackrel{\mu}{\curvearrowright} \mathbb{R})=H^{1}(\mathfrak{a n} ; \mathfrak{a n} \stackrel{\mu}{\curvearrowright} \mathbb{R}) .
$$

COROLLARY 9. Let $G$ be a connected semisimple Lie group with finite center of real rank at least 3 whose simple factors are of real rank at least 2 , and let $\Gamma$ be an irreducible cocompact lattice in $G$. Let $G=K A N$ be an Iwasawa decomposition. Then the action $\Gamma \backslash G \curvearrowleft A N$ by right multiplication is parameter rigid.

Proof. Under the assumptions of this corollary, (2) in Lemma 7 follows from the case $\lambda=0$ in Corollary 11 below, and (3) in Lemma 7 follows from Theorem 8. This implies parameter rigidity of the action.

But this does not cover the case of real rank 2. In this case we only know vanishing of the cohomology with coefficients corresponding to restricted roots.

THEOREM 10. Let $G$ be a connected semisimple Lie group with finite center of real rank at least 2 with neither compact factors nor simple factors locally isomorphic to $\mathrm{SO}_{0}(n, 1)(n \geq 2)$ or $\mathrm{SU}(n, 1)(n \geq 2)$, and let $\Gamma$ be an irreducible cocompact lattice in G. Fix a Cartan decomposition $\mathfrak{g}=\mathfrak{k} \oplus \mathfrak{p}$ and a maximal abelian subspace $\mathfrak{a}$ of $\mathfrak{p}$ with the associated restricted root system $\Sigma$. Let $\mathcal{F}_{A}$ be the orbit foliation of the action $\Gamma \backslash G \curvearrowleft A$ by right multiplication. Then we have:

(1) (Katok and Spatzier [12, Theorem 3.6])

$$
H^{1}\left(\mathcal{F}_{A}\right)=H^{1}(\mathfrak{a})
$$

(2) (Kanai $[10$, Theorem 2.2])

$$
H^{1}\left(\mathcal{F}_{A} ; \mathfrak{a} \stackrel{\lambda}{\curvearrowright} \mathbb{R}\right)=H^{1}(\mathfrak{a} ; \mathfrak{a} \stackrel{\lambda}{\curvearrowright} \mathbb{R})=0
$$

for any $\lambda \in \Sigma$.

Remark. In [10, Theorem 2.2(2)] it is written that $u$ (which is notation from [10]) is $C^{\infty}$ if the conditions (i) and (ii) from that paper are satisfied, but those conditions (i) and (ii) are always satisfied, so that we get the above result.

COROLLARY 11. Let $G$ be a connected semisimple Lie group with finite center of real rank at least 2 with neither compact factors nor simple factors locally isomorphic to 
$\mathrm{SO}_{0}(n, 1)(n \geq 2)$ or $\mathrm{SU}(n, 1)(n \geq 2)$, and let $\Gamma$ be an irreducible cocompact lattice in G. Fix a Cartan decomposition $\mathfrak{g}=\mathfrak{k} \oplus \mathfrak{p}$, a maximal abelian subspace $\mathfrak{a}$ of $\mathfrak{p}$ with the associated restricted root system $\Sigma$, and a positive system $\Sigma_{+}$of $\Sigma$. Let $\mathcal{F}$ be the orbit foliation of the action $\Gamma \backslash G \curvearrowleft A N$ by right multiplication. Then we have

$$
H^{1}(\mathcal{F} ; \mathfrak{a n} \stackrel{\lambda}{\curvearrowright} \mathbb{R})=H^{1}(\mathfrak{a n} ; \mathfrak{a n} \stackrel{\lambda}{\curvearrowright} \mathbb{R})
$$

for any $\lambda \in \Sigma \cup\{0\}$, where $\lambda: \mathfrak{a} \rightarrow \mathbb{R}$ is regarded as $\lambda: \mathfrak{a n} \rightarrow \mathbb{R}$ by extending it as 0 on $\mathfrak{n}$.

Proof. Let $[\omega] \in H^{1}(\mathcal{F} ; \mathfrak{a n} \stackrel{\lambda}{\curvearrowright} \mathbb{R})$; that is, $d_{\mathcal{F}} \omega+\lambda \omega_{0} \wedge \omega=0$, where $\omega_{0}$ is the canonical 1-form of $\Gamma \backslash G \curvearrowleft A N$. By restriction to $T \mathcal{F}_{A}$ we get $d_{\mathcal{F}_{A}} \omega+\lambda \omega_{0} \wedge \omega=0$. Note that $\omega_{0}$ restricts to the canonical 1-form of $\Gamma \backslash G \curvearrowleft A$. So

$$
[\omega] \in H^{1}\left(\mathcal{F}_{A} ; \mathfrak{a} \stackrel{\lambda}{\curvearrowright} \mathbb{R}\right)=H^{1}(\mathfrak{a} ; \mathfrak{a} \stackrel{\lambda}{\curvearrowright} \mathbb{R}) .
$$

There exist a linear map $\phi: \mathfrak{a} \rightarrow \mathbb{R}$ such that $\lambda(H) \phi\left(H^{\prime}\right)-\lambda\left(H^{\prime}\right) \phi(H)=0$ for all $H$, $H^{\prime} \in \mathfrak{a}$ and a $C^{\infty}$ function $h: \Gamma \backslash G \rightarrow \mathbb{R}$ satisfying $\omega=\phi \omega_{0}+d_{\mathcal{F}_{A}} h+\lambda \omega_{0} h$. For any $H \in \mathfrak{a}$ and $X \in \mathfrak{g}_{\mu}$ for $\mu \in \Sigma_{+}$,

$$
\begin{aligned}
0 & =H \omega(X)-X \omega(H)-\mu(H) \omega(X)+\lambda(H) \omega(X) \\
& =H \omega(X)-X(\phi(H)+H h+\lambda(H) h)-\mu(H) \omega(X)+\lambda(H) \omega(X) \\
& =H \omega(X)-H X h-[X, H] h-\lambda(H) X h-\mu(H) \omega(X)+\lambda(H) \omega(X) \\
& =H(\omega(X)-X h)+(\lambda(H)-\mu(H))(\omega(X)-X h) .
\end{aligned}
$$

If $\mu \neq \lambda$, take $H_{0} \in \mathfrak{a}$ such that $\lambda\left(H_{0}\right)-\mu\left(H_{0}\right) \neq 0$. Then the above equation for $H=H_{0}$ and the boundedness of $\omega(X)-X h$ imply $\omega(X)-X h=0$. If $\mu=\lambda$, take $H \neq 0$. We can apply Moore's ergodicity theorem since $G$ has finite center and no compact factor and $\Gamma$ is irreducible. So the flow $e^{t H}(t \in \mathbb{R})$ has a dense orbit and $\omega(X)-X h=\psi(X)$ for some $\psi(X) \in \mathbb{R}$. Let $\omega^{\prime}=\omega-d_{\mathcal{F}} h-\lambda \omega_{0} h$. Then

$$
\begin{aligned}
& \omega^{\prime}(H)=\phi(H) \quad \text { for } H \in \mathfrak{a}, \\
& \omega^{\prime}(X)= \begin{cases}0 & \text { for } X \in \mathfrak{g}_{\mu} \text { and } \mu \neq \lambda, \\
\psi(X) & \text { for } X \in \mathfrak{g}_{\lambda} \text { and } \lambda \in \Sigma_{+} .\end{cases}
\end{aligned}
$$

Therefore, $[\omega]=\left[\omega^{\prime}\right] \in H^{1}(\mathfrak{a n} ; \mathfrak{a n} \stackrel{\lambda}{\curvearrowright} \mathbb{R})$.

By Corollary 11, the proof of Theorem 2 reduces to that of the following proposition.

Proposition 12. Let $G$ be a connected semisimple Lie group. Fix a Cartan decomposition $\mathfrak{g}=\mathfrak{k} \oplus \mathfrak{p}$, a maximal abelian subspace $\mathfrak{a}$ of $\mathfrak{p}$ with the associated restricted root system $\Sigma$, and a positive system $\Sigma_{+}$of $\Sigma$. Let $G=K A N$ be the corresponding Iwasawa decomposition. Let $M \stackrel{\rho_{0}}{\curvearrowleft} A N$ be a $C^{\infty}$ locally free action on a closed manifold $M$ with the orbit foliation $\mathcal{F}$. If

$$
H^{1}(\mathcal{F})=H^{1}(\mathfrak{a n})
$$


and

$$
H^{1}(\mathcal{F} ; \mathfrak{a n} \stackrel{\lambda}{\curvearrowright} \mathbb{R})=H^{1}(\mathfrak{a n} ; \mathfrak{a n} \stackrel{\lambda}{\curvearrowright} \mathbb{R})
$$

for all $\lambda \in \Sigma_{+}$, where $\lambda: \mathfrak{a} \rightarrow \mathbb{R}$ is regarded as $\lambda: \mathfrak{a n} \rightarrow \mathbb{R}$ by extending it linearly as 0 on $\mathfrak{n}$, then $\rho_{0}$ is parameter rigid.

Note that we need no assumption on the simple factors of $G$ in this proposition.

Remark. In Theorem 10 we assume that

$G$ has no simple factors locally isomorphic to $\mathrm{SO}_{0}(n, 1)(n \geq 2)$ or $\mathrm{SU}(n, 1)(n \geq 2)$. (*)

If (1) in Theorem 10 is true without the assumption $(*)$, then (2) in Theorem 10 and Corollary 11 are true without the assumption $(*)$. Hence Theorem 2 will be true without the assumption $(*)$ by Proposition 12 .

\section{Proof of Proposition 12}

To prove Proposition 12, it suffices to show that $\left.\lambda \circ \varphi_{\rho}\right|_{\mathfrak{a}} \in \Sigma_{+}$for any $\lambda \in \Sigma_{+}$and any $\rho \in \mathcal{A}(\mathcal{F}, A N)$, by Lemma 7. At this moment we know only that $\left.\varphi_{\rho}\right|_{\mathfrak{a}}$ is an element of $\operatorname{GL}(\mathfrak{a})$, so it is not clear whether $\left.\varphi_{\rho}\right|_{\mathfrak{a}}$ preserves $\Sigma_{+}$. To prove it we need rigidity of quasi-isometries of symmetric spaces.

For the proof of Proposition 12 we may assume that $G$ has no compact factors, since this does not change $A N$. Recall that $\operatorname{Inn}(\mathfrak{g})=\operatorname{Ad}(G)=G / Z(G)$, where $Z(G)$ denotes the center of $G$, and $G / Z(G)$ has trivial center. Replacing $G$ with $G / Z(G)$ also does not change $A N$, so we may assume $G=\operatorname{Inn}(\mathfrak{g})$ as well.

The mapping an $\mapsto$ an $K$ gives a canonical diffeomorphism $A N \simeq G / K$ by the Iwasawa decomposition. Henceforth we identify $A N$ with $G / K$ in this way. This is $A N$-equivariant.

Recall that the identification $\mathfrak{p} \simeq T_{K} G / K$ is by $\left.X \mapsto(d / d t) e^{t X} K\right|_{t=0}$. In the following, $K$ denotes the subgroup $K$ or the point $K$ in $G / K$, depending on the context. $G$-invariant Riemannian metrics on $G / K$ are in one-to-one correspondence with inner products on $\mathfrak{p}$ invariant under $K \stackrel{\text { Ad }}{\curvearrowright} \mathfrak{p}$. We equip $G / K$ with a $G$-invariant Riemannian metric $g$ corresponding to the restriction of $B_{\theta}$ to $\mathfrak{p}$, where $\theta$ is the Cartan involution associated with the Cartan decomposition $\mathfrak{g}=\mathfrak{k} \oplus \mathfrak{p}, B$ is the Killing form of $\mathfrak{g}$, and $B_{\theta}(X, Y)=$ $-B(X, \theta Y)$ for $X, Y \in \mathfrak{g}$. The restriction of $B_{\theta}$ to $\mathfrak{p}$ is the same as the restriction of $B$ to $\mathfrak{p}$. We give $A N$ the Riemannian metric which makes the identification $A N \simeq G / K$ an isometry. This Riemannian metric is $A N$-invariant. Geodesics in $G / K$ passing $K$ at time 0 are of the form $e^{t X} K(t \in \mathbb{R})$ for $X \in \mathfrak{p}$. Note that $e^{t X} K(t \in \mathbb{R})$ for $X \in \mathfrak{g} \backslash \mathfrak{p}$ is not a geodesic in general. In $A N$, curves of the form $n e^{t H}(t \in \mathbb{R})$ for fixed $n \in N$ and $H \in \mathfrak{a}$ are geodesics.

The decomposition $\mathfrak{g}=\mathfrak{k} \oplus \mathfrak{p}$ is orthogonal with respect to the positive definite symmetric bilinear form $B_{\theta}$. Let $\mathfrak{g}_{\lambda}^{\prime}$ be the orthogonal projection to $\mathfrak{p}$ with respect to $\mathfrak{g}=$ $\mathfrak{k} \oplus \mathfrak{p}$ of $\mathfrak{g}_{\lambda}$ for $\lambda \in \Sigma$. The space $\mathfrak{g}_{\lambda}^{\prime}$ has the same dimension as $\mathfrak{g}_{\lambda}$ since $\mathfrak{k}=\operatorname{ker}(\theta-\mathrm{id})$ and $\theta \mathfrak{g}_{\lambda}=\mathfrak{g}_{-\lambda}$. This orthogonal projection maps $\mathfrak{a n}$ isomorphically to $\mathfrak{p}$ by the Iwasawa decomposition $\mathfrak{g}=\mathfrak{k} \oplus \mathfrak{a} \oplus \mathfrak{n}$. Therefore,

$$
\mathfrak{p}=\mathfrak{a} \oplus \mathfrak{n}^{\prime} \quad \text { where } \quad \mathfrak{n}^{\prime}=\bigoplus_{\lambda \in \Sigma_{+}} \mathfrak{g}_{\lambda}^{\prime} .
$$


Note that $\mathfrak{a} \perp \mathfrak{n}^{\prime}$ since $\mathfrak{a} \perp \mathfrak{g}_{\lambda}$ for $\lambda \in \Sigma$ and $\mathfrak{a} \perp \mathfrak{k}$ with respect to $B_{\theta}$. Observe that the differentiation

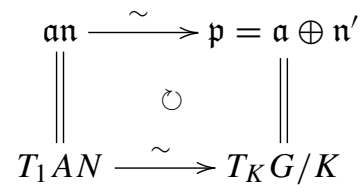

at 1 of the identification $A N \simeq G / K$ maps an to $\mathfrak{p}$ by the orthogonal projection with respect to $\mathfrak{g}=\mathfrak{k} \oplus \mathfrak{p}$. Therefore, $\mathfrak{a}$ maps identically to $\mathfrak{a}$ and $\mathfrak{n}$ maps isomorphically to $\mathfrak{n}^{\prime}$. So $\mathfrak{a} \perp \mathfrak{n}$ in $\mathfrak{a n}$.

For any $\rho \in \mathcal{A}(\mathcal{F}, A N)$ and $x \in M$, consider the diagram

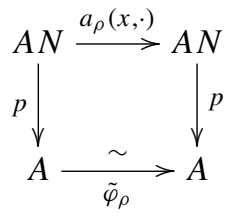

where $p$ is the natural projection. We give $A$ a left-invariant Riemannian metric for which the restriction $\mathfrak{a} \rightarrow \mathfrak{a}$ of the natural projection $\mathfrak{a n} \rightarrow \mathfrak{a}$ to $\mathfrak{n}^{\perp}=\mathfrak{a}$ becomes an isometry; i.e. we consider the restriction of $B$ to $\mathfrak{a}$. Then $p$ is a distance-respecting projection by Proposition 5 and $a_{\rho}(x, \cdot)$ is a fiber-respecting bi-Lipschitz diffeomorphism over $\tilde{\varphi}_{\rho}$ by Proposition 6.

Since $G=\operatorname{Ad}(G)$, we have $G=G_{1} \times \cdots \times G_{\ell}$, where $G_{i}$ is a connected non-compact simple Lie group with trivial center. Since any two maximal compact subgroups of $G$ are conjugate by an inner automorphism of $G$, we have $K=K_{1} \times \cdots \times$ $K_{\ell}$, where $K_{i}$ is a maximal compact subgroup of $G_{i}$, and $G / K=G_{1} / K_{1} \times \cdots \times G_{\ell} / K_{\ell}$. Let $\mathfrak{g}_{i}=\mathfrak{k}_{i} \oplus \mathfrak{p}_{i}$ be the Cartan decomposition. Then $\mathfrak{p}=\mathfrak{p}_{1} \oplus \cdots \oplus \mathfrak{p}_{\ell}$. Let $g_{i}$ be the $G_{i}$-invariant Riemannian metric on $G_{i} / K_{i}$ corresponding to the restriction of the Killing form $B_{i}$ of $\mathfrak{g}_{i}$ to $\mathfrak{p}_{i}$. Since

$$
B\left(\left(X_{1}, \ldots, X_{\ell}\right),\left(Y_{1}, \ldots, Y_{\ell}\right)\right)=B_{1}\left(X_{1}, Y_{1}\right)+\cdots+B_{\ell}\left(X_{\ell}, Y_{\ell}\right)
$$

for $X_{i}, Y_{i} \in \mathfrak{g}_{i}$, we have $g=g_{1} \times \cdots \times g_{\ell}$. Since maximal abelian subspaces in $\mathfrak{p}$ are conjugate by $\operatorname{Ad}(k)$ for some $k \in K$ and $\operatorname{Ad}(k)$ preserves each $\mathfrak{p}_{i}$, we have $\mathfrak{a}=\mathfrak{a}_{1} \oplus \cdots \oplus$ $\mathfrak{a}_{\ell}$ for some maximal abelian subspace $\mathfrak{a}_{i}$ of $\mathfrak{p}_{i}$. Let

$$
\mathfrak{g}_{i}=\mathfrak{a}_{i} \oplus \mathfrak{m}_{i} \oplus \underset{\lambda_{i} \in \Sigma_{i}}{\bigoplus}\left(\mathfrak{g}_{i}\right)_{\lambda_{i}}
$$

be the restricted root space decomposition of $\mathfrak{g}_{i}$. Then

$$
\mathfrak{g}=\bigoplus_{i=1}^{\ell} \mathfrak{a}_{i} \oplus \bigoplus_{i=1}^{\ell} \mathfrak{m}_{i} \oplus \bigoplus_{i=1}^{\ell} \bigoplus_{\lambda_{i} \in \Sigma_{i}}\left(\mathfrak{g}_{i}\right)_{\lambda_{i}}
$$

is the restricted root space decomposition of $\mathfrak{g}$. Thus $\Sigma=\Sigma_{1} \cup \cdots \cup \Sigma_{\ell}$, where $\lambda_{i}: \mathfrak{a}_{i} \rightarrow$ $\mathbb{R}$ in $\Sigma_{i}$ is regarded as $\lambda_{i}: \mathfrak{a} \rightarrow \mathbb{R}$ in $\Sigma$ by extending it linearly on $\mathfrak{a}_{j}(j \neq i)$ as 0 . Hence $\mathfrak{g}_{\lambda_{i}}=\left(\mathfrak{g}_{i}\right)_{\lambda_{i}}$ for $\lambda_{i} \in \Sigma_{i}$. Since any two simple systems of $\Sigma$ are conjugate by $\operatorname{Ad}(k)$ for 
some $k \in N_{K}(\mathfrak{a})=N_{K_{1}}\left(\mathfrak{a}_{1}\right) \times \cdots \times N_{K_{\ell}}\left(\mathfrak{a}_{\ell}\right)$, it follows that $\Sigma_{+}=\Sigma_{1+} \cup \cdots \cup \Sigma_{\ell+}$ for some positive system $\Sigma_{i+}$ of $\Sigma_{i}$. Hence $\mathfrak{n}=\mathfrak{n}_{1} \oplus \cdots \oplus \mathfrak{n}_{\ell}$, where $\mathfrak{n}_{i}=\bigoplus_{\lambda_{i} \in \Sigma_{i+}}\left(\mathfrak{g}_{i}\right)_{\lambda_{i}}$. Of course we also have

$$
A=A_{1} \times \cdots \times A_{\ell}, \quad N=N_{1} \times \cdots \times N_{\ell}, \quad A N=A_{1} N_{1} \times \cdots \times A_{\ell} N_{\ell} .
$$

The metric $g$ on $A N$ decomposes as $g=g_{1} \times \cdots \times g_{\ell}$, where $g_{i}$ on $A_{i} N_{i}$ is defined by the identification $A_{i} N_{i} \simeq G_{i} / K_{i}, a_{i} n_{i} \mapsto a_{i} n_{i} K_{i}$. The same kind of decomposition holds for the metric on $A$.

The map $a_{\rho}(x, \cdot): G / K \rightarrow G / K$ is a quasi-isometry. By Kleiner and Leeb [13, Theorem 1.1.2] there exist a permutation $\sigma \in \mathfrak{S}_{\ell}$ and quasi-isometries

$$
\Phi_{i}:\left(G_{i} / K_{i}, g_{i}\right) \rightarrow\left(G_{\sigma(i)} / K_{\sigma(i)}, g_{\sigma(i)}\right)
$$

such that $a_{\rho}(x, \cdot)$ and

$$
\Phi:\left(x_{1}, \ldots, x_{\ell}\right) \mapsto\left(\Phi_{\sigma^{-1}(1)}\left(x_{\sigma^{-1}(1)}\right), \ldots, \Phi_{\sigma^{-1}(\ell)}\left(x_{\sigma^{-1}(\ell)}\right)\right)
$$

are close. Then

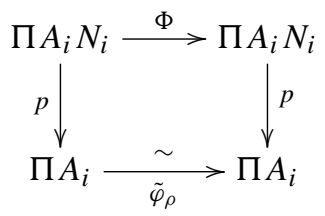

is fiber-respecting. In fact, let $C>0$ be a constant such that

$$
d_{\mathcal{H}}\left(a_{\rho}(x, a N), \tilde{\varphi}_{\rho}(a) N\right)<C
$$

for all $a \in A$, and let $C^{\prime}>0$ be such that

$$
d\left(\Phi(s), a_{\rho}(x, s)\right)<C^{\prime}
$$

for all $s \in A N$; then we have

$$
\begin{aligned}
d_{\mathcal{H}}\left(\Phi(a N), \tilde{\varphi}_{\rho}(a) N\right) & \leq d_{\mathcal{H}}\left(\Phi(a N), a_{\rho}(x, a N)\right)+d_{\mathcal{H}}\left(a_{\rho}(x, a N), \tilde{\varphi}_{\rho}(a) N\right) \\
& <C^{\prime}+C
\end{aligned}
$$

for all $a \in A$.

LEMMA 13. There exist linear isomorphisms $\varphi_{i}: \mathfrak{a}_{i} \rightarrow \mathfrak{a}_{\sigma(i)}$ such that

$$
\tilde{\varphi}_{\rho}\left(a_{1}, \ldots, a_{\ell}\right)=\left(\tilde{\varphi}_{\sigma^{-1}(1)}\left(a_{\sigma^{-1}(1)}\right), \ldots, \tilde{\varphi}_{\sigma^{-1}(\ell)}\left(a_{\sigma^{-1}(\ell)}\right)\right)
$$

for all $a_{i} \in A_{i}$ and

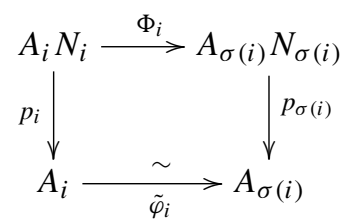

is fiber-respecting, where $p_{i}$ is the natural projection and $\tilde{\varphi}_{i}$ is the isomorphism with differential $\varphi_{i}$. 
Proof. Let $\tilde{\varphi}_{\rho}\left(a_{1}, \ldots, a_{\ell}\right)=\left(\tilde{\phi}_{1}\left(a_{1}, \ldots, a_{\ell}\right), \ldots, \tilde{\phi}_{\ell}\left(a_{1}, \ldots, a_{\ell}\right)\right)$ for $a_{i} \in A_{i}$. For fixed $i$ and for any $H_{i} \in \mathfrak{a}_{i}$ and $t \in \mathbb{R}$, the Hausdorff distance between

$$
\begin{aligned}
\Phi & \left(p^{-1}\left(1, \ldots, 1, e^{t H_{i}}, 1, \ldots, 1\right)\right) \\
& =\Phi\left(N_{1} \times \cdots \times N_{i-1} \times e^{t H_{i}} N_{i} \times N_{i+1} \times \cdots \times N_{\ell}\right) \\
& =\Phi_{\sigma^{-1}(1)}\left(N_{\sigma^{-1}(1)}\right) \times \cdots \times \Phi_{i}\left(e^{t H_{i}} N_{i}\right) \times \cdots \times \Phi_{\sigma^{-1}(\ell)}\left(N_{\sigma^{-1}(\ell)}\right)
\end{aligned}
$$

and

$$
\begin{aligned}
& \tilde{\phi}_{1}\left(1, \ldots, e^{t H_{i}}, \ldots, 1\right) N_{1} \times \cdots \times \tilde{\phi}_{\ell}\left(1, \ldots, e^{t H_{i}}, \ldots, 1\right) N_{\ell} \\
& \quad=e^{t \phi_{1}\left(0, \ldots, H_{i}, \ldots, 0\right)} N_{1} \times \cdots \times e^{t \phi_{\ell}\left(0, \ldots, H_{i}, \ldots, 0\right)} N_{\ell}
\end{aligned}
$$

is bounded by a constant $C>0$, where $\phi_{j}$ is the differential of $\tilde{\phi}_{j}$. Thus

$$
d_{\mathcal{H}}\left(\Phi_{\sigma^{-1}(j)}\left(N_{\sigma^{-1}(j)}\right), e^{t \phi_{j}\left(0, \ldots, H_{i}, \ldots, 0\right)} N_{j}\right)<C
$$

for $j \neq \sigma(i)$. Hence

$$
d\left(e^{t \phi_{j}\left(0, \ldots, H_{i}, \ldots, 0\right)}, 1\right)=d_{\mathcal{H}}\left(e^{t \phi_{j}\left(0, \ldots, H_{i}, \ldots, 0\right)} N_{j}, N_{j}\right)<2 C
$$

for all $t \in \mathbb{R}$, which implies $\phi_{j}\left(0, \ldots, H_{i}, \ldots, 0\right)=0$. Therefore,

$$
\begin{aligned}
\tilde{\varphi}_{\rho}\left(e^{H_{1}}, \ldots, e^{H_{\ell}}\right) & =\left(\tilde{\phi}_{1}\left(e^{\left(H_{1}, \ldots, H_{\ell}\right)}\right), \ldots, \tilde{\phi}_{\ell}\left(e^{\left(H_{1}, \ldots, H_{\ell}\right)}\right)\right) \\
& =\left(e^{\phi_{1}\left(0, \ldots, H_{\sigma^{-1}(1)}, \ldots, 0\right)}, \ldots, e^{\phi_{\ell}\left(0, \ldots, H_{\sigma^{-1}(\ell)}, \ldots, 0\right)}\right) \\
& =\left(\tilde{\phi}_{1}\left(e^{H_{\sigma^{-1}}(1)}\right), \ldots, \tilde{\phi}_{\ell}\left(e^{H_{\sigma^{-1}(\ell)}}\right)\right) \\
& =\left(\tilde{\varphi}_{\sigma^{-1}(1)}\left(e^{H_{\sigma^{-1}(1)}}\right), \ldots, \tilde{\varphi}_{\sigma^{-1}(\ell)}\left(e^{H_{\sigma^{-1}(\ell)}}\right)\right),
\end{aligned}
$$

where we put $\tilde{\varphi}_{j}=\tilde{\phi}_{\sigma(j)}: A_{j} \rightarrow A_{\sigma(j)}$. Finally, by looking at $\sigma(i)$ th components of (4) and (5), we have

$$
d_{\mathcal{H}}\left(\Phi_{i}\left(e^{t H_{i}} N_{i}\right), \tilde{\varphi}_{i}\left(e^{t H_{i}}\right) N_{\sigma(i)}\right)=d_{\mathcal{H}}\left(\Phi_{i}\left(e^{t H_{i}} N_{i}\right), e^{t \phi_{\sigma(i)}\left(0, \ldots, H_{i}, \ldots, 0\right)} N_{\sigma(i)}\right)<C ;
$$

hence $\Phi_{i}$ is fiber-respecting over $\tilde{\varphi}_{i}$.

Therefore, Proposition 12 follows if $\lambda \circ \varphi_{\sigma^{-1}(i)} \in \Sigma_{\sigma^{-1}(i)+}$ for all $\lambda \in \Sigma_{i+}$, since $\Sigma_{+}=$ $\Sigma_{1+} \cup \cdots \cup \Sigma_{\ell+}$ and $\left.\lambda \circ \varphi_{\rho}\right|_{\mathfrak{a}}=\lambda \circ \varphi_{\sigma^{-1}(i)}$ for $\lambda \in \Sigma_{i+}$.

Since $G_{\sigma(i)} / K_{\sigma(i)}$ and $G_{i} / K_{i}$ are quasi-isometric, $\mathfrak{g}_{\sigma(i)}$ and $\mathfrak{g}_{i}$ are isomorphic. Fix an isomorphism $\alpha: \mathfrak{g}_{\sigma(i)} \simeq \mathfrak{g}_{i}$ such that

$$
\alpha\left(\mathfrak{k}_{\sigma(i)}\right)=\mathfrak{k}_{i}, \quad \alpha\left(\mathfrak{p}_{\sigma(i)}\right)=\mathfrak{p}_{i}, \quad \alpha\left(\mathfrak{a}_{\sigma(i)}\right)=\mathfrak{a}_{i}
$$

and $\alpha$ takes $\Sigma_{\sigma(i)+}$ to $\Sigma_{i+}$. Then $\alpha$ canonically induces isomorphisms

$$
\mathfrak{n}_{\sigma(i)} \simeq \mathfrak{n}_{i}, \quad G_{\sigma(i)} \simeq G_{i}, \quad K_{\sigma(i)} \simeq K_{i}, \quad A_{\sigma(i)} \simeq A_{i}, \quad N_{\sigma(i)} \simeq N_{i}
$$

and isometries

$$
\left(G_{\sigma(i)} / K_{\sigma(i)}, g_{\sigma(i)}\right) \simeq\left(G_{i} / K_{i}, g_{i}\right), \quad A_{\sigma(i)} N_{\sigma(i)} \simeq A_{i} N_{i} .
$$


In this way we identify $A_{\sigma(i)} N_{\sigma(i)}$ with $A_{i} N_{i}$ etc. Hence now

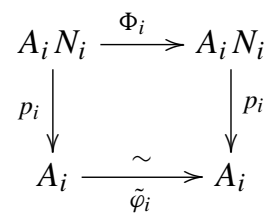

is fiber-respecting, and to complete the proof of Proposition 12 it suffices to show $\lambda \circ \varphi_{i} \in$ $\Sigma_{i+}$ for any $\lambda \in \Sigma_{i+}$.

We consider the following two cases separately.

- The group $G_{i}$ is of real rank at least 2 or is locally isomorphic to $\operatorname{Sp}(n, 1)(n \geq 2)$ or $F_{4}^{-20}$.

- The group $G_{i}$ is of real rank 1 .

We can treat $G_{i}$ locally isomorphic to $\operatorname{Sp}(n, 1)(n \geq 2)$ or $F_{4}^{-20}$ in either case.

From now on we will no longer consider the original objects $G, K, A, N, g, \Sigma, \varphi_{\rho}$ etc; we will focus only on the decomposed objects $G_{i}, K_{i}, A_{i}, N_{i}, g_{i}, \Sigma_{i}, \varphi_{i}$ etc. Hence we will drop all the subscripts $i$ to simplify the notation. So we have

$$
G, \mathfrak{g}, K, \mathfrak{k}, \mathfrak{p}, \theta, B, A, \mathfrak{a}, N, \mathfrak{n}, \mathfrak{n}^{\prime}, \Sigma, \Sigma_{+}, \mathfrak{g}_{\lambda}, \mathfrak{g}_{\lambda}^{\prime}, g, \varphi, \tilde{\varphi}, \Phi, p
$$

but we do not have $M, \rho$ and $a_{\rho}$. Recall that $G=\operatorname{Ad}(G), g$ is the restriction of $B$ at $T_{K} G / K=\mathfrak{p}, A N$ is equipped with a Riemannian metric by the identification $A N \simeq G / K$, the Riemannian metric of $A$ is the one which makes $\left.p_{*}\right|_{\mathfrak{a}}: \mathfrak{a} \simeq \mathfrak{a}$ an isometry, and

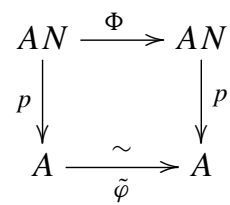

is fiber-respecting. Under these conditions we must prove $\lambda \circ \varphi \in \Sigma_{+}$for any $\lambda \in \Sigma_{+}$.

5.1. The case where $G$ is of real rank at least 2 or is locally isomorphic to $\operatorname{Sp}(n, 1)(n \geq 2)$ or $F_{4}^{-20}$. By Kleiner and Leeb [13, Theorem 1.1.3] for $G$ of real rank at least 2 and by Pansu [18, 1 . Théorème] for $G$ locally isomorphic to $\operatorname{Sp}(n, 1)(n \geq 2)$ or $F_{4}^{-20}$, there exists a homothety

$$
F:(G / K, g) \rightarrow(G / K, g)
$$

close to $\Phi$. Thus there is a constant $c>0$ such that $g\left(F_{*} X, F_{*} Y\right)=\operatorname{cg}(X, Y)$ for all $x \in G / K$ and $X, Y \in T_{x} G / K$, so $F:(G / K, c g) \rightarrow(G / K, g)$ is an isometry. Since the isometry group of $G / K$ acts transitively, there exists the minimum $K_{0} \in(-\infty, 0)$ of the sectional curvature of $(G / K, g)$. Then $c K_{0}$ is the minimum of the sectional curvature of $(G / K, c g)$. Since they are isometric we must have $K_{0}=c K_{0}$; hence $c=1$. Thus $F:(G / K, g) \rightarrow(G / K, g)$ is an isometry. 
Since $F$ is close to $\Phi$,

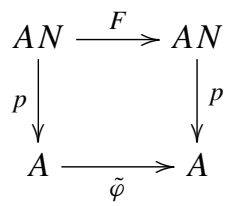

is fiber-respecting.

Let $F(1)^{-1}=a_{0} n_{0} \in A N$. We have

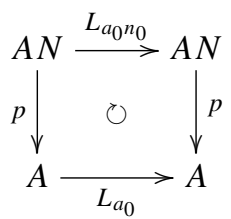

where $L$ denotes left multiplication. Since $L_{a_{0} n_{0}}$ is an isometry,

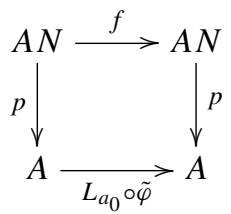

is fiber-respecting, where $f=L_{a_{0} n_{0}} \circ F$. Since $L_{a_{0}} \circ \tilde{\varphi}$ and $\tilde{\varphi}$ are close,

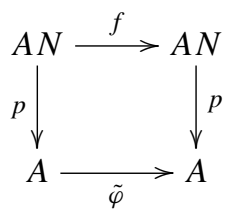

is also fiber-respecting. Note that $f$ is an isometry and $f(1)=1$.

LEMMA 14. The map $\tilde{\varphi}$ is an isometry.

Proof. There exists a constant $C>0$ such that $d_{\mathcal{H}}(f(a N), \tilde{\varphi}(a) N)<C$ for all $a \in A$. Then we have

$$
\begin{aligned}
|d(1, a)-d(1, \tilde{\varphi}(a))|= & \left|d_{\mathcal{H}}(f(N), f(a N))-d_{\mathcal{H}}(N, \tilde{\varphi}(a) N)\right| \\
\leq & \left|d_{\mathcal{H}}(f(N), f(a N))-d_{\mathcal{H}}(f(N), \tilde{\varphi}(a) N)\right| \\
& +\left|d_{\mathcal{H}}(f(N), \tilde{\varphi}(a) N)-d_{\mathcal{H}}(N, \tilde{\varphi}(a) N)\right| \\
\leq & d_{\mathcal{H}}(f(a N), \tilde{\varphi}(a) N)+d_{\mathcal{H}}(f(N), N) \\
< & 2 C
\end{aligned}
$$

for all $a \in A$. Hence for all $t>0$ and $H \in \mathfrak{a}$ we have

$$
\left|d\left(1, e^{t H}\right)-d\left(1, e^{t \varphi H}\right)\right|<2 C ;
$$


that is,

$$
|t\|H\|-t\|\varphi H\||<2 C
$$

This implies

$$
\|\varphi H\|=\|H\| .
$$

Thus $\tilde{\varphi}$ is an isometry.

Now we regard $f$ as $f: G / K \rightarrow G / K$ and $p: G / K \rightarrow A$. Consider

$$
f_{*}: \mathfrak{p}=T_{K} G / K \rightarrow \mathfrak{p}=T_{K} G / K .
$$

LEMMA 15. We have $f_{*}(\mathfrak{a})=\mathfrak{a}$ and $\left.f_{*}\right|_{\mathfrak{a}}=\varphi: \mathfrak{a} \rightarrow \mathfrak{a}$.

Proof. Take any $H \in \mathfrak{a}$. Let $f_{*} H=X+Y$ for some $X \in \mathfrak{a}$ and $Y \in \mathfrak{n}^{\prime}$. Since

$$
\|H\|^{2}=\left\|f_{*} H\right\|^{2}=\|X\|^{2}+\|Y\|^{2} \geq\|X\|^{2},
$$

we have $\|H\| \geq\|X\|$. Because $e^{t H} K(t \in \mathbb{R})$ is a geodesic and $f$ is an isometry, $f\left(e^{t H} K\right)(t \in \mathbb{R})$ is also a geodesic and $f\left(e^{t H} K\right)=e^{t X+t Y} K$. Let $t>0$.

Since $f$ is fiber-respecting over $\tilde{\varphi}$, there exists a constant $C>0$ such that

$$
d_{\mathcal{H}}\left(f\left(p^{-1}\left(e^{t H}\right)\right), p^{-1}\left(\tilde{\varphi}\left(e^{t H}\right)\right)\right)<C .
$$

Since $e^{t X+t Y} K=f\left(e^{t H} K\right) \in f\left(p^{-1}\left(e^{t H}\right)\right)$ and by the definition of the Hausdorff distance, there exists $x \in p^{-1}\left(\tilde{\varphi}\left(e^{t H}\right)\right)$ such that $d\left(e^{t X+t Y} K, x\right)<C$.

The map $p$ is distance-decreasing, since $d\left(a, a^{\prime}\right)=d\left(p^{-1}(a), p^{-1}\left(a^{\prime}\right)\right)$ for all $a, a^{\prime} \in$ A. So

$$
d\left(e^{t X}, \tilde{\varphi}\left(e^{t H}\right)\right)=d\left(p\left(e^{t X+t Y} K\right), p(x)\right) \leq d\left(e^{t X+t Y} K, x\right)<C .
$$

Since $\tilde{\varphi}$ is an isometry,

$$
d\left(\tilde{\varphi}\left(e^{t H}\right), 1\right)=d\left(e^{t H}, 1\right)=t\|H\| .
$$

By the triangle inequality we have

$$
t\|H\|-t\|X\|=d\left(1, \tilde{\varphi}\left(e^{t H}\right)\right)-d\left(1, e^{t X}\right) \leq d\left(e^{t X}, \tilde{\varphi}\left(e^{t H}\right)\right)<C
$$

for all $t>0$. This forces $\|H\|=\|X\|$, and then $Y=0$ by the equation $\|H\|^{2}=\|X\|^{2}+$ $\|Y\|^{2}$. Hence $f_{*} H=X \in \mathfrak{a}$.

For the second assertion we have by (6) that

$$
d\left(e^{t f_{*} H}, e^{t \varphi H}\right)<C
$$

for any $t \in \mathbb{R}$. This implies $f_{*} H=\varphi H$.

Proposition 16. Let $\mathfrak{g}$ be a real semisimple Lie algebra and let $G=\operatorname{Inn}(\mathfrak{g})$. (Recall that the Lie algebra of $G$ is naturally isomorphic to $\mathfrak{g}$, and $G$ is the identity component of Aut(g).) Fix a maximal compact subgroup $K$ of $G$.

(1) Let $\psi \in \operatorname{Aut}(\mathfrak{g})$ and consider $\Psi \in \operatorname{Aut}(G)$ defined by $\Psi(g)=\psi g \psi^{-1}$. The automorphism $\Psi$ permutes the maximal compact subgroups of $G$. Identifying the set of 
all maximal compact subgroups of $G$ with $G / K$ by $\mathrm{g} \mathrm{Kg}^{-1} \leftrightarrow \mathrm{g} K$, we have that the map $I_{\psi}: G / K \rightarrow G / K$ induced by $\Psi$ is an isometry with respect to the $G$-invariant Riemannian metric defined by the restriction of the Killing form to the orthogonal complement of the Lie algebra of $K$.

(2) Suppose $\mathfrak{g}$ has no compact simple factor. Then the mapping $\psi \mapsto I_{\psi}$ is an isomorphism from $\operatorname{Aut}(\mathfrak{g})$ to $\operatorname{Isom}(G / K)$.

Proof. This is Exercise 7 in Helgason [7, Ch. VI]. A proof can be found in Solutions to Exercises.

By Proposition 16 there exists $\psi \in \operatorname{Aut}(\mathfrak{g})$ such that $f=I_{\psi}$. Since $f(K)=K$, we have $\Psi(K)=K$. This implies

$$
f(g K)=\Psi(g) K
$$

for all $g \in G$. We have $\psi(\mathfrak{k})=\mathfrak{k}$. Since

$$
\mathfrak{p}=\{X \in \mathfrak{g} \mid B(X, Y)=0 \text { for all } Y \in \mathfrak{k}\}
$$

and $B$ is $\psi$-invariant, we also have $\psi(\mathfrak{p})=\mathfrak{p}$. Hence $f_{*}=\left.\psi\right|_{\mathfrak{p}}: \mathfrak{p} \rightarrow \mathfrak{p}$ by (7) and $\psi(\mathfrak{a})=\mathfrak{a}$ by Lemma 15 . Therefore, $\left.\psi\right|_{\mathfrak{a}}=\varphi: \mathfrak{a} \rightarrow \mathfrak{a}$ again by Lemma 15 . Since $\psi$ is an isomorphism of $\mathfrak{g}$ which preserves $\mathfrak{a}$, we have $\psi^{-1} \mathfrak{g}_{\lambda}=\mathfrak{g}_{\left.\lambda \circ \psi\right|_{\mathfrak{a}}}$ for any $\lambda \in \Sigma$. Thus $\lambda \circ \varphi=\left.\lambda \circ \psi\right|_{\mathfrak{a}} \in \Sigma$ if $\lambda \in \Sigma$. We must show that $\lambda \circ \varphi=\left.\lambda \circ \psi\right|_{\mathfrak{a}} \in \Sigma_{+}$if $\lambda \in \Sigma_{+}$.

For a Weyl chamber $C$ in $\mathfrak{a}$, let

$$
\Sigma_{C}=\{\lambda \in \Sigma \mid \lambda(H)>0 \text { for some } H \in C\}
$$

be the positive system corresponding to $C$, let $\mathfrak{n}_{C}=\bigoplus_{\lambda \in \Sigma_{C}} \mathfrak{g}_{\lambda}$, and let $N_{C}$ be the Lie subgroup corresponding to $\mathfrak{n}_{C}$.

Let $C_{0} \subset \mathfrak{a}$ be the Weyl chamber corresponding to $\Sigma_{+}$, i.e.

$$
C_{0}=\left\{H \in \mathfrak{a} \mid \lambda(H)>0 \text { for all } \lambda \in \Sigma_{+}\right\} .
$$

Then $C_{1}=\psi C_{0}$ is a Weyl chamber in $\mathfrak{a}$. We have $\lambda \in \Sigma_{+}$if and only if $\lambda \circ\left(\left.\psi\right|_{\mathfrak{a}}\right)^{-1} \in$ $\Sigma_{C_{1}}$. Thus $\psi \mathfrak{n}=\mathfrak{n}_{C_{1}}$. By (7) we have $f(N K)=N_{C_{1}} K$. Therefore, the Hausdorff distance between $N_{C_{1}} K$ and $N K$ is finite.

LEMMA 17. If $C$ and $C^{\prime}$ are distinct Weyl chambers in $\mathfrak{a}$, then the Hausdorff distance between $N_{C} K$ and $N_{C^{\prime}} K$ is infinite.

Proof. Take $\lambda \in \Sigma_{C} \backslash \Sigma_{C^{\prime}}$. Hence $\mathfrak{g}_{\lambda} \subset \mathfrak{n}_{C}$ and $\mathfrak{g}_{-\lambda} \subset \mathfrak{n}_{C^{\prime}}$. We will prove that $e^{\mathfrak{g}-\lambda} K$ contains points arbitrarily far from $N_{C} K$. Let $H_{\lambda} \in \mathfrak{a}$ be the element defined by $\lambda(H)=$ $B\left(H_{\lambda}, H\right)$ for all $H \in \mathfrak{a}$. By Knapp [14, Proposition 6.52] there exists non-zero $X_{\lambda} \in \mathfrak{g}_{\lambda}$ such that:

- $\left[X_{\lambda}, \theta X_{\lambda}\right]=B\left(X_{\lambda}, \theta X_{\lambda}\right) H_{\lambda}$;

- $B\left(X_{\lambda}, \theta X_{\lambda}\right)=-2 / B\left(H_{\lambda}, H_{\lambda}\right)<0$; 
- the subspace $\mathbb{R} \theta X_{\lambda} \oplus \mathbb{R} H_{\lambda} \oplus \mathbb{R} X_{\lambda}$ is a Lie subalgebra of $\mathfrak{g}$ isomorphic to $\mathfrak{s l}(2, \mathbb{R})$.

The isomorphism is given by

$$
\begin{aligned}
X_{-\lambda}^{\prime}=\theta X_{\lambda} & \longleftrightarrow\left(\begin{array}{ll}
0 & 0 \\
1 & 0
\end{array}\right), \\
H_{\lambda}^{\prime}=\frac{2}{B\left(H_{\lambda}, H_{\lambda}\right)} H_{\lambda} & \longleftrightarrow\left(\begin{array}{cc}
1 & 0 \\
0 & -1
\end{array}\right), \\
X_{\lambda}^{\prime}=-X_{\lambda} & \longleftrightarrow\left(\begin{array}{ll}
0 & 1 \\
0 & 0
\end{array}\right) .
\end{aligned}
$$

For any $x \in \mathbb{R}$ we have

$$
\left(\begin{array}{ll}
1 & 0 \\
x & 1
\end{array}\right)=\left(\begin{array}{cc}
1 & \frac{x}{1+x^{2}} \\
0 & 1
\end{array}\right)\left(\begin{array}{cc}
\frac{1}{\sqrt{1+x^{2}}} & 0 \\
0 & \sqrt{1+x^{2}}
\end{array}\right)\left(\begin{array}{cc}
\frac{1}{\sqrt{1+x^{2}}} & \frac{x}{\sqrt{1+x^{2}}} \\
-\frac{x}{\sqrt{1+x^{2}}} & \frac{1}{\sqrt{1+x^{2}}}
\end{array}\right)
$$

This can be regarded as an equation of elements in the universal cover $\widetilde{\mathrm{SL}}(2, \mathbb{R})$ of $\operatorname{SL}(2, \mathbb{R})$. We rewrite it using the exponential map:

$$
\begin{aligned}
\exp \left(\begin{array}{ll}
0 & 0 \\
x & 0
\end{array}\right)= & \exp \left(\begin{array}{cc}
0 & \frac{x}{1+x^{2}} \\
0 & 0
\end{array}\right) \exp \left(\begin{array}{cc}
-\frac{\log \left(1+x^{2}\right)}{2} & 0 \\
0 & \frac{\log \left(1+x^{2}\right)}{2}
\end{array}\right) \\
& \cdot \exp \left(\begin{array}{cc}
0 & -\arctan x \\
\arctan x & 0
\end{array}\right) .
\end{aligned}
$$

Mapping the above equation by the homomorphism $\widetilde{\mathrm{SL}}(2, \mathbb{R}) \rightarrow G$, we get

$$
\begin{aligned}
\exp \left(x X_{-\lambda}^{\prime}\right)= & \exp \left(\frac{x}{1+x^{2}} X_{\lambda}^{\prime}\right) \exp \left(-\frac{\log \left(1+x^{2}\right)}{2} H_{\lambda}^{\prime}\right) \\
& \cdot \exp \left(\arctan x\left(X_{-\lambda}^{\prime}-X_{\lambda}^{\prime}\right)\right) .
\end{aligned}
$$

Note that

$$
\begin{gathered}
\exp \left(x X_{-\lambda}^{\prime}\right) \in e^{\mathfrak{g}-\lambda} \subset N_{C^{\prime}}, \quad \exp \left(\frac{x}{1+x^{2}} X_{\lambda}^{\prime}\right) \in e^{\mathfrak{g}_{\lambda}} \subset N_{C}, \\
\exp \left(-\frac{\log \left(1+x^{2}\right)}{2} H_{\lambda}^{\prime}\right) \in A
\end{gathered}
$$

Since $\theta\left(X_{-\lambda}^{\prime}-X_{\lambda}^{\prime}\right)=X_{-\lambda}^{\prime}-X_{\lambda}^{\prime}$, we have $X_{-\lambda}^{\prime}-X_{\lambda}^{\prime} \in \mathfrak{k}$; hence

$$
\exp \left(\arctan x\left(X_{-\lambda}^{\prime}-X_{\lambda}^{\prime}\right)\right) \in K \text {. }
$$

Thus (8) gives the Iwasawa decomposition of $\exp \left(x X_{-\lambda}^{\prime}\right)$ as an element of $G=N_{C} A K$. Therefore,

$$
\begin{aligned}
d\left(\exp \left(x X_{-\lambda}^{\prime}\right) K, N_{C} K\right) & =d\left(\exp \left(\frac{x}{1+x^{2}} X_{\lambda}^{\prime}\right) \exp \left(-\frac{\log \left(1+x^{2}\right)}{2} H_{\lambda}^{\prime}\right) K, N_{C} K\right) \\
& =d\left(\exp \left(-\frac{\log \left(1+x^{2}\right)}{2} H_{\lambda}^{\prime}\right) K, N_{C} K\right)
\end{aligned}
$$




$$
\begin{aligned}
& =d\left(\exp \left(-\frac{\log \left(1+x^{2}\right)}{2} H_{\lambda}^{\prime}\right) K, K\right) \\
& =\left\|-\frac{\log \left(1+x^{2}\right)}{2} H_{\lambda}^{\prime}\right\| \\
& =\frac{\log \left(1+x^{2}\right)}{\sqrt{B\left(H_{\lambda}, H_{\lambda}\right)}} .
\end{aligned}
$$

This shows that $N_{C^{\prime}} K$ contains points arbitrarily far from $N_{C} K$.

Thus $C_{1}=C_{0}$ and so $\psi \mathfrak{n}=\mathfrak{n}$. Hence $\lambda \circ \varphi=\left.\lambda \circ \psi\right|_{\mathfrak{a}} \in \Sigma_{+}$if $\lambda \in \Sigma_{+}$.

\subsection{The case where $G$ is of real rank 1}

PROPOSITION 18. If

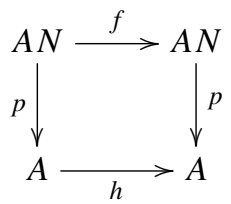

is fiber-respecting, $f$ is a quasi-isometry and $h$ is a map, then $h$ is close to the identity map.

The map $\tilde{\varphi}$ is close to the identity map by this proposition. But since $\tilde{\varphi}$ is a homomorphism, $\tilde{\varphi}$ must be the identity map. Hence $\lambda \circ \varphi=\lambda \in \Sigma_{+}$for all $\lambda \in \Sigma_{+}$, and this concludes the proof of Proposition 12.

Proposition 18 is Farb and Mosher [5, Proposition 5.8] when $G$ is locally isomorphic to $\mathrm{SO}_{0}(n, 1)$. For the other cases it is basically Reiter Ahlin [19, Theorem 33], but the proof there seems incomplete. To get the conclusion of Proposition 18 we need to argue at some point in the same manner as Farb and Mosher do. Here we give a proof of Proposition 18 following the arguments by Farb and Mosher and Reiter Ahlin.

We have $\Sigma_{+}=\{\lambda\}$ for $G$ locally isomorphic to $\operatorname{SO}_{0}(n, 1)$ and $\Sigma_{+}=\{\lambda, 2 \lambda\}$ for the other cases. Accordingly $\mathfrak{n}=\mathfrak{g}_{\lambda}$ in the former case and $\mathfrak{n}=\mathfrak{g}_{\lambda} \oplus \mathfrak{g}_{2 \lambda}$ in the latter case. Take $H \in \mathfrak{a}$ such that $\lambda(H)=1$. Hence $\mathfrak{a}=\mathbb{R} H$. We identify $A$ with $\mathbb{R}$ by $e^{t H} \rightarrow t$.

We write the proof for the case of $\Sigma_{+}=\{\lambda, 2 \lambda\}$, but no change is needed when we have $\Sigma_{+}=\{\lambda\}$ except a notational one.

Let $g_{t}$ be the Riemannian metric on $N$ induced from $g$ by the embedding $N \hookrightarrow$ $A N, x \mapsto x e^{t H}$. Let $d$ and $d_{t}$ be the metrics induced from $g$ and $g_{t}$ respectively. Since $x\left(y e^{t H}\right)=(x y) e^{t H}$, i.e. the embedding $N \hookrightarrow A N$ is $N$-equivariant, $g_{t}$ is a left-invariant Riemannian metric on $N$. Let $\|\cdot\|_{j}$ be a norm on $\mathfrak{g}_{j \lambda}(j=1,2)$ and set $|x|=\max \left\{\|\xi\|_{1},\|v\|_{2}^{\frac{1}{2}}\right\}$ for $x \in N$, where $\log x=\xi+v$ for $\xi \in \mathfrak{g}_{\lambda}, v \in \mathfrak{g}_{2 \lambda}$. Let $\phi_{t}: N \rightarrow N$ be the map defined by $\phi_{t}(x)=e^{t H} x e^{-t H}$. Then

$$
\begin{aligned}
\left|\phi_{t}(x)\right| & =\left|e^{t H} e^{\xi+v} e^{-t H}\right|=\left|\exp \left(e^{t \text { ad } H}(\xi+v)\right)\right| \\
& =\left|\exp \left(e^{t} \xi+e^{2 t} v\right)\right|=\max \left\{e^{t}\|\xi\|_{1}, e^{t}\|v\|_{2}^{\frac{1}{2}}\right\}
\end{aligned}
$$




$$
\begin{aligned}
& =e^{t} \max \left\{\|\xi\|_{1},\|v\|_{2}^{\frac{1}{2}}\right\}=e^{t}\left|e^{\xi+v}\right| \\
& =e^{t}|x|
\end{aligned}
$$

for any $x \in N$ and $t \in \mathbb{R}$.

LEMMA 19. There exists $K_{1} \geq 1$ such that

$$
\frac{1}{K_{1}} e^{-t}\left|x^{-1} y\right|-K_{1} \leq d_{t}(x, y) \leq K_{1} e^{-t}\left|x^{-1} y\right|+K_{1}
$$

for all $t \in \mathbb{R}$ and $x, y \in N$.

Proof. Since $e^{t H} x=\phi_{t}(x) e^{t H}, \phi_{t}:\left(N, g_{0}\right) \rightarrow\left(N, g_{t}\right)$ is an isometry. Hence

$$
d_{t}(x, y)=d_{t}\left(1, x^{-1} y\right)=d_{0}\left(1, \phi_{-t}\left(x^{-1} y\right)\right) .
$$

It is known that there exists a constant $K_{1} \geq 1$ such that

$$
\frac{1}{K_{1}}|x|-K_{1} \leq d_{0}(1, x) \leq K_{1}|x|+K_{1}
$$

for all $x \in N$. See for example Breuillard [3, Proposition 4.5]. Therefore,

$$
d_{t}(x, y) \leq K_{1}\left|\phi_{-t}\left(x^{-1} y\right)\right|+K_{1}=K_{1} e^{-t}\left|x^{-1} y\right|+K_{1}
$$

and

$$
\frac{1}{K_{1}} e^{-t}\left|x^{-1} y\right|-K_{1}=\frac{1}{K_{1}}\left|\phi_{-t}\left(x^{-1} y\right)\right|-K_{1} \leq d_{t}(x, y) .
$$

COROLLARY 20. There exists $K_{2} \geq 1$ such that for any fixed $t_{0} \in \mathbb{R}$ we have

$$
\frac{1}{K_{2}^{2}} e^{t_{0}-t} \leq \frac{d_{t}(x, y)}{d_{t_{0}}(x, y)} \leq K_{2}^{2} e^{t_{0}-t}
$$

if $t \leq t_{0}$ and $\left|x^{-1} y\right|>\left(K_{1}^{2}+1\right) e^{t_{0}}$.

Proof. If $t \leq t_{0}$ and $\left|x^{-1} y\right|>\left(K_{1}^{2}+1\right) e^{t_{0}}$, then we have $e^{-t}\left|x^{-1} y\right|>K_{1}^{2}+1$, and hence

$$
\left(\frac{1}{K_{1}}-\frac{K_{1}}{K_{1}^{2}+1}\right) e^{-t}\left|x^{-1} y\right| \leq d_{t}(x, y) \leq\left(K_{1}+\frac{K_{1}}{K_{1}^{2}+1}\right) e^{-t}\left|x^{-1} y\right|
$$

by Lemma 19. Since

$$
\frac{1}{K_{1}}-\frac{K_{1}}{K_{1}^{2}+1}>0
$$

there exists $K_{2} \geq 1$, which is independent of $t_{0}$, such that

$$
\frac{1}{K_{2}} e^{-t}\left|x^{-1} y\right| \leq d_{t}(x, y) \leq K_{2} e^{-t}\left|x^{-1} y\right|
$$

under the above conditions. In particular

$$
\frac{1}{K_{2}} e^{-t_{0}}\left|x^{-1} y\right| \leq d_{t_{0}}(x, y) \leq K_{2} e^{-t_{0}}\left|x^{-1} y\right| .
$$

We get the conclusion from these two inequalities. 
A map $\sigma: S \rightarrow X$ between geodesic spaces is called uniformly proper if there exist constants $K \geq 1, C \geq 0$ and a function $\rho: \mathbb{R}_{\geq 0} \rightarrow \mathbb{R}_{\geq 0}$ with $\lim _{a \rightarrow \infty} \rho(a)=\infty$ such that

$$
\rho(d(x, y)) \leq d(\sigma(x), \sigma(y)) \leq K d(x, y)+C
$$

for all $x, y \in S$. We call $\rho, K$ and $C$ the uniformity data for $\sigma$.

LEMMA 21. The embedding $\left(N, d_{t}\right) \hookrightarrow(A N, d)$ is uniformly proper for each $t \in \mathbb{R}$, and the uniformity data are independent of $t$. In fact there exists a function $\rho: \mathbb{R}_{\geq 0} \rightarrow \mathbb{R}_{\geq 0}$ with $\lim _{a \rightarrow \infty} \rho(a)=\infty$ such that

$$
\rho\left(d_{t}(x, y)\right) \leq d\left(x e^{t H}, y e^{t H}\right) \leq d_{t}(x, y)
$$

for all $x, y \in N$ and $t \in \mathbb{R}$.

Proof. The second inequality is obvious. For the first inequality, define $\rho_{1}: \mathbb{R}_{\geq 0} \rightarrow \mathbb{R}_{\geq 0}$ by

$$
\rho_{1}(R)=\sup \left\{d_{0}(1, x) \mid x \in N, d(1, x)=R\right\} .
$$

Then $\rho_{1}$ is strictly increasing and $\lim _{R \rightarrow \infty} \rho_{1}(R)=\infty$. We have $d_{0}(1, x) \leq \rho_{1}(d(1, x))$ for any $x \in N$; hence $d_{0}(x, y) \leq \rho_{1}(d(x, y))$ for all $x, y \in N$. Since

$$
\begin{aligned}
d_{t}(x, y) & =d_{0}\left(e^{-t H} x e^{t H}, e^{-t H} y e^{t H}\right) \\
& \leq \rho_{1}\left(d\left(e^{-t H} x e^{t H}, e^{-t H} y e^{t H}\right)\right) \\
& =\rho_{1}\left(d\left(x e^{t H}, y e^{t H}\right)\right),
\end{aligned}
$$

we get $\rho_{1}^{-1}\left(d_{t}(x, y)\right) \leq d\left(x e^{t H}, y e^{t H}\right)$. So $\rho=\rho_{1}^{-1}$ satisfies the required properties.

Lemma 22. Let $X, Y, S, T$ be geodesic spaces, let $f: X \rightarrow Y$ be a quasi-isometry, and let $\sigma: S \rightarrow X, \tau: T \rightarrow Y$ be uniformly proper maps such that $d_{\mathcal{H}}(f \sigma(S), \tau(T))<\infty$. Take any map $g: S \rightarrow T$ satisfying $\sup _{x \in S} d(f \sigma(x), \tau g(x))<\infty$. Then $g$ is a quasi-isometry and the quasi-isometry constants depend only on the quasi-isometry constants for $f$, the uniformity data for $\sigma$ and $\tau$, and $\sup _{x \in S} d(f \sigma(x), \tau g(x))$.

Proof. This is Farb and Mosher [5, Lemma 2.1].

We identify $h: A \rightarrow A$ with $h: \mathbb{R} \rightarrow \mathbb{R}$ by $h\left(e^{t H}\right)=e^{h(t) H}$. Define $f_{t}:\left(N, d_{t}\right) \rightarrow$ $\left(N, d_{h(t)}\right)$ by $f\left(x e^{t H}\right)=f_{t}(x) e^{u(x, t) H}$. Then $f_{t}$ satisfies the property of Lemma 22. In fact since $f$ is fiber-respecting over $h$, there exists a constant $C_{1}>0$ such that $d_{\mathcal{H}}\left(f\left(p^{-1}\left(e^{t H}\right)\right), p^{-1}\left(e^{h(t) H}\right)\right)<C_{1}$ for all $t \in \mathbb{R}$. Hence there exists $y \in N$ such that $d\left(f\left(x e^{t H}\right), y e^{h(t) H}\right)<C_{1}$. Therefore,

$$
\begin{aligned}
d\left(f\left(x e^{t H}\right), f_{t}(x) e^{h(t) H}\right) & =d\left(f_{t}(x) e^{u(x, t) H}, f_{t}(x) e^{h(t) H}\right) \\
& \leq d\left(f_{t}(x) e^{u(x, t) H}, y e^{h(t) H}\right)<C_{1}
\end{aligned}
$$

for all $x \in N$ and $t \in \mathbb{R}$. By Lemma 21 and Lemma 22, $f_{t}:\left(N, d_{t}\right) \rightarrow\left(N, d_{h(t)}\right)$ is a quasi-isometry with quasi-isometry constants independent of $t$. 
Let $\partial A N$ be the Gromov boundary of $A N$. Then $\partial A N=\{\infty\} \cup N$. The quasi-isometry $f$ induces a map $\partial f: \partial A N \rightarrow \partial A N$.

LEMMA 23. $\partial f(\infty)=\infty$.

Proof. Assume the contrary: $\partial f(\infty)=x \in N$. Take $y \in N$ with $y \neq(\partial f)^{-1}(\infty)$. Let $\gamma$ be the directed geodesic connecting $y$ and $\infty$. Then the Hausdorff distance between $f(\gamma)$ and the directed geodesic $\gamma^{\prime}$ connecting $\partial f(y)$ and $x$ is finite. Hence the height of $f(\gamma)$ is bounded above. Since $h$ is a quasi-isometry, we can choose $t_{0} \in \mathbb{R}$ so that $h\left(t_{0}\right)$ is as large as we wish. Therefore, the height of $f\left(p^{-1}\left(e^{t_{0} H}\right)\right)$ is also large. But we always have $f\left(y e^{t_{0} H}\right) \in f\left(p^{-1}\left(e^{t_{0} H}\right)\right) \cap f(\gamma) \neq \varnothing$, which is impossible.

For any $x \in N, x e^{t H}(t \in \mathbb{R})$ is a geodesic of $A N$ connecting $x \in \partial A N$ and $\infty$. Then $f\left(x e^{t H}\right)(t \in \mathbb{R})$ is a quasigeodesic of $A N$. By Lemma 23 there exists a constant $C_{2}>0$ such that $d_{\mathcal{H}}\left(f\left(x e^{\mathbb{R} H}\right), \partial f(x) e^{\mathbb{R} H}\right)<C_{2}$. By (9)

$$
|u(x, t)-h(t)|\|H\|<C_{1} .
$$

There exists $s(x, t) \in \mathbb{R}$ such that $d\left(f\left(x e^{t H}\right), \partial f(x) e^{s(x, t) H}\right)<C_{2}$. We have

$$
\begin{aligned}
|u(x, t)-s(x, t)|\|H\| & =d\left(\partial f(x) e^{u(x, t) H}, \partial f(x) e^{s(x, t) H}\right) \\
& =d\left(p^{-1}\left(e^{u(x, t) H}\right), \partial f(x) e^{s(x, t) H}\right) \\
& \leq d\left(f\left(x e^{t H}\right), \partial f(x) e^{s(x, t) H}\right)<C_{2} .
\end{aligned}
$$

By (10) and (11) we get

$$
|s(x, t)-h(t)|\|H\|<C_{1}+C_{2} .
$$

Therefore,

$$
\begin{aligned}
d( & \left.f_{t}(x) e^{h(t) H}, \partial f(x) e^{h(t) H}\right) \\
\leq & d\left(f_{t}(x) e^{h(t) H}, f_{t}(x) e^{u(x, t) H}\right)+d\left(f_{t}(x) e^{u(x, t) H}, \partial f(x) e^{s(x, t) H}\right) \\
& \quad+d\left(\partial f(x) e^{s(x, t) H}, \partial f(x) e^{h(t) H}\right) \\
< & |u(x, t)-h(t)|\|H\|+C_{2}+|s(x, t)-h(t)|\|H\| \\
< & 2 C_{1}+2 C_{2} .
\end{aligned}
$$

Hence

$$
d_{h(t)}\left(f_{t}(x), \partial f(x)\right) \leq \rho^{-1}\left(d\left(f_{t}(x) e^{h(t) H}, \partial f(x) e^{h(t) H}\right)\right)<\rho^{-1}\left(2 C_{1}+2 C_{2}\right) .
$$

That is, $f_{t}$ and $\partial f$ are close, and the constant of closeness is independent of $t$. Thus $\partial f:\left(N, d_{t}\right) \rightarrow\left(N, d_{h(t)}\right)$ is a quasi-isometry with constants independent of $t$, so there exists a constant $K_{3} \geq 1$ such that

$$
\frac{1}{K_{3}} d_{t}(x, y)-K_{3} \leq d_{h(t)}(\partial f(x), \partial f(y)) \leq K_{3} d_{t}(x, y)+K_{3}
$$

for all $x, y \in N$ and $t \in \mathbb{R}$. 
LEMma 24. For any fixed $t_{0} \in \mathbb{R}$ we have

$$
\frac{1}{2 K_{3}} d_{t}(x, y) \leq d_{h(t)}(\partial f(x), \partial f(y)) \leq 2 K_{3} d_{t}(x, y)
$$

for all $t \leq t_{0}$ and $x, y \in N$ with $\left|x^{-1} y\right|>e^{t_{0}} K_{1}\left(2 K_{3}^{2}+K_{1}\right)$.

Proof. If $t \leq t_{0}$ and $\left|x^{-1} y\right|>e^{t_{0}} K_{1}\left(2 K_{3}^{2}+K_{1}\right)$, we have

$$
d_{t}(x, y) \geq \frac{1}{K_{1}} e^{-t}\left|x^{-1} y\right|-K_{1} \geq 2 K_{3}^{2} .
$$

Hence

$$
d_{h(t)}(\partial f(x), \partial f(y)) \leq K_{3} d_{t}(x, y)+K_{3} \leq 2 K_{3} d_{t}(x, y)
$$

and

$$
d_{h(t)}(\partial f(x), \partial f(y)) \geq \frac{1}{K_{3}} d_{t}(x, y)-\frac{1}{2 K_{3}} 2 K_{3}^{2} \geq \frac{1}{2 K_{3}} d_{t}(x, y) .
$$

It is easy to show that $h$ is a quasi-isometry of $\mathbb{R}$. See Farb and Mosher [5, Lemma 5.1].

LEMMA 25. There exists $L>0$ such that for any $t, t_{0} \in \mathbb{R}$ with $t+L \leq t_{0}$ we have $h(t) \leq$ $h\left(t_{0}\right)$.

Proof. Recall that $h$ is close to $s(x, \cdot)$ as we saw in (12). By the definition of $s(x, t)$ we see $s(x, t) \rightarrow \pm \infty$ as $t \rightarrow \pm \infty$. So $h(t) \rightarrow \pm \infty$ as $t \rightarrow \pm \infty$. Let $K \geq 1$ be a constant such that $(1 / K)|s-t|-K \leq|h(s)-h(t)| \leq K|s-t|+K$ for all $s, t \in \mathbb{R}$. Take $L=4 K^{2}$ and assume the contrary; i.e. suppose there were $s_{0}, t_{0} \in \mathbb{R}$ with $s_{0}+L \leq t_{0}$ such that $h\left(t_{0}\right) \leq h\left(s_{0}\right)$. We have $\left|h\left(s_{0}\right)-h\left(t_{0}\right)\right| \geq 3 K$ and $\left|h\left(s_{0}\right)-h(t)\right| \geq 3 K$ for any $t \geq t_{0}$. For $t_{0} \leq t \leq t_{0}+1$ we have $\left|h(t)-h\left(t_{0}\right)\right| \leq 2 K$. Hence we must have $h(t) \leq h\left(s_{0}\right)$ for all $t_{0} \leq t \leq t_{0}+1$. Now we have $s_{0}+L \leq t_{0}+1$ and $h\left(t_{0}+1\right) \leq h\left(s_{0}\right)$. Hence this time we get $h(t) \leq h\left(s_{0}\right)$ for all $t_{0}+1 \leq t \leq t_{0}+2$. By repeating we see that $h(t) \leq h\left(s_{0}\right)$ for all $t \geq t_{0}$, which is a contradiction.

LEMMA 26. For any fixed $t_{0} \in \mathbb{R}$, we have

$$
\frac{1}{K_{2}^{2}} e^{h\left(t_{0}\right)-h(t)} \leq \frac{d_{h(t)}(\partial f(x), \partial f(y))}{d_{h\left(t_{0}\right)}(\partial f(x), \partial f(y))} \leq K_{2}^{2} e^{h\left(t_{0}\right)-h(t)}
$$

if $t \leq t_{0}-L$ and

$$
\left|x^{-1} y\right|>K_{1}^{2} K_{3} e^{-h(0)}\left(\frac{1}{K_{1} e^{-h(0)}}\left(\frac{K_{1}}{K_{3}}+K_{3}+K_{1}\right)+\left(K_{1}^{2}+1\right) e^{h\left(t_{0}\right)}\right) .
$$

Proof. If $t \leq t_{0}-L$ and

$$
\left|x^{-1} y\right|>K_{1}^{2} K_{3} e^{-h(0)}\left(\frac{1}{K_{1} e^{-h(0)}}\left(\frac{K_{1}}{K_{3}}+K_{3}+K_{1}\right)+\left(K_{1}^{2}+1\right) e^{h\left(t_{0}\right)}\right),
$$


then

$$
\begin{aligned}
\left|\partial f(x)^{-1} \partial f(y)\right| & \geq \frac{1}{K_{1} e^{-h(0)}}\left(d_{h(0)}(\partial f(x), \partial f(y))-K_{1}\right) \\
& \geq \frac{1}{K_{1} e^{-h(0)}}\left(\frac{1}{K_{3}} d_{0}(x, y)-K_{3}-K_{1}\right) \\
& \geq \frac{1}{K_{1} e^{-h(0)}}\left(\frac{1}{K_{3}}\left(\frac{1}{K_{1}}\left|x^{-1} y\right|-K_{1}\right)-K_{3}-K_{1}\right) \\
& =\frac{1}{K_{1}^{2} K_{3} e^{-h(0)}}\left|x^{-1} y\right|-\frac{1}{K_{1} e^{-h(0)}}\left(\frac{K_{1}}{K_{3}}+K_{3}+K_{1}\right) \\
& >\left(K_{1}^{2}+1\right) e^{h\left(t_{0}\right)}
\end{aligned}
$$

and $h(t) \leq h\left(t_{0}\right)$. So we get the desired inequality by Corollary 20 .

LEMMA 27. There exists $C_{3}>0$ such that for any $t_{0} \in \mathbb{R}$ and $t \leq t_{0}$, we have

$$
h(t) \geq t-t_{0}+h\left(t_{0}\right)-C_{3} .
$$

Proof. Fix $t_{0}$ and take $x, y \in N$ with $\left|x^{-1} y\right|$ large enough so that we can apply Corollary 20, Lemma 24 and Lemma 26. Then for any $t \leq t_{0}-L$, we have

$$
\begin{aligned}
\frac{1}{2 K_{2}^{2} K_{3}} e^{h\left(t_{0}\right)-h(t)} d_{t_{0}}(x, y) & \leq \frac{1}{K_{2}^{2}} e^{h\left(t_{0}\right)-h(t)} d_{h\left(t_{0}\right)}(\partial f(x), \partial f(y)) \\
& \leq d_{h(t)}(\partial f(x), \partial f(y)) \\
& \leq 2 K_{3} d_{t}(x, y) \\
& \leq 2 K_{2}^{2} K_{3} e^{t_{0}-t} d_{t_{0}}(x, y) .
\end{aligned}
$$

Hence

$$
e^{h\left(t_{0}\right)-h(t)} \leq 4 K_{2}^{4} K_{3}^{2} e^{t_{0}-t}
$$

Taking log we get

$$
h\left(t_{0}\right)-h(t) \leq t_{0}-t+\log \left(4 K_{2}^{4} K_{3}^{2}\right) .
$$

Since $h$ is a quasi-isometry, $h\left(t_{0}\right)-h(t)-t_{0}+t$ is bounded above for $t_{0}-L \leq t \leq t_{0}$ by a constant independent of $t_{0}$. Hence the claim is proved.

Let $\bar{f}: A N \rightarrow A N$ be a coarse inverse of $f$; i.e. $\bar{f}$ is a quasi-isometry such that $\bar{f} \circ f$ and $f \circ \bar{f}$ are close to the identity map. Let $\bar{h}: \mathbb{R} \rightarrow \mathbb{R}$ be a coarse inverse of $h$. It is easy to show that $\bar{f}$ is fiber-respecting over $\bar{h}$. Apply Lemma 27 to $\bar{f}$ and $\bar{h}$ rather than $f$ and $h$. Then there exists $C_{3}^{\prime}>0$ such that

$$
\bar{h}(s) \geq s-s_{0}+\bar{h}\left(s_{0}\right)-C_{3}^{\prime}
$$

for all $s \leq s_{0}$. Now we can argue completely in the same way as in Farb and Mosher (see p. 167 just after Claim 5.9 in [5]) to prove that $h$ is close to the identity map. 


\section{Necessary conditions for parameter rigidity}

From this section forward we consider necessary conditions for parameter rigidity. (For the definition of parameter rigidity, see the beginning of $\S 2$.) These necessary conditions are given by a certain vanishing of zeroth and first cohomology of the orbit foliation. The main results are Theorem 20 and Theorem 32.

Let $M \stackrel{\rho_{0}}{\curvearrowleft} S$ denote a $C^{\infty}$ locally free action of a connected simply connected solvable Lie group $S$ on a closed $C^{\infty}$ manifold $M$, with the orbit foliation $\mathcal{F}$.

Recall that a connected simply connected solvable Lie group $S$ is called of exponential type if the exponential map exp: $\mathfrak{s} \rightarrow S$ is a diffeomorphism, or equivalently, every eigenvalue of ad $X$ either is 0 or has non-zero real part for each $X \in \mathfrak{s}$. For a proof of this equivalence, see Dixmier [4, Théorème 3] or Saito [20]. A derivation of a Lie algebra is called an outer derivation if it is not an inner derivation.

The first necessary condition is the following.

THEOREM 28. (Vanishing of $H^{0}$ ) Assume that $S$ is of exponential type and there is an outer derivation of $\mathfrak{s}$. If $M \stackrel{\rho_{0}}{\curvearrowleft} S$ is parameter rigid, then $M$ is connected and $H^{0}(\mathcal{F})=$ $H^{0}(\mathfrak{s})$.

We will prove Theorem 28 in $§ 7$.

COROLLARY 29. Let $N \neq 1$ be a connected simply connected nilpotent Lie group and let $M \stackrel{\rho_{0}}{\curvearrowleft} N$ be a parameter rigid action. Then $M$ is connected and $H^{0}(\mathcal{F})=H^{0}(\mathfrak{n})$.

Proof. Every non-zero nilpotent Lie algebra over any field has an outer derivation. See Jacobson [9].

Note that $H^{0}(\mathcal{F})$ consists of real-valued leafwise constant $C^{\infty}$ functions on $M$, and $H^{0}(\mathfrak{s})$ (as a subspace of $H^{0}(\mathcal{F})$ ) consists of real-valued constant functions on $M$. Hence we have $H^{0}(\mathcal{F})=H^{0}(\mathfrak{s})$ if and only if leafwise constant $C^{\infty}$ functions are constant. This is satisfied if there is a dense leaf of $\mathcal{F}$. In the proof of Theorem 28 we do not prove the existence of a dense leaf of $\mathcal{F}$. We prove $H^{0}(\mathcal{F})=H^{0}(\mathfrak{s})$ somewhat algebraically, without studying dynamical properties of the foliation $\mathcal{F}$.

Remark. The author does not know whether Theorem 28 remains true if we drop one of the two assumptions on $S$. One possibility of constructing counterexamples which are parameter rigid but where $H^{0}(\mathcal{F})$ is huge is the following. Take a connected simply connected solvable Lie group $S$ and a cocompact lattice $\Gamma$ in $S$ such that the following hold.

- $\quad S$ has no outer automorphisms.

- $\quad \Gamma$ is a rigid lattice in $S$, which means that if $\Gamma^{\prime}$ is a lattice in $S$ and $\alpha: \Gamma \rightarrow \Gamma^{\prime}$ is an isomorphism, then $\alpha$ extends to an automorphism of $S$. (This terminology is taken from Starkov [21].)

The author does not know whether such $S$ and $\Gamma$ exist. But if we have such a pair, Maruhashi [17, Proposition 4] says that the action $\Gamma \backslash S \curvearrowleft S$ defined by right multiplication is parameter rigid, because in this case parameter rigidity is equivalent to the rigidity 
of the lattice $\Gamma$. Then the action $S^{1} \times \Gamma \backslash S \curvearrowleft S$ defined by $(x, y) s=(x, y s)$ is perhaps parameter rigid by the first condition, whereas $H^{0}(\mathcal{F})$ is now identified with the space of all real-valued $C^{\infty}$ functions on $S^{1}$.

Recall the following theorem.

THEOREM 21. (Maruhashi [16]) Let $N$ be a connected simply connected nilpotent Lie group, and let $M \stackrel{\rho_{0}}{\curvearrowleft} N$ be a $C^{\infty}$ locally free action. Then the following are equivalent.

- $\quad$ The action $\rho_{0}$ is parameter rigid and $H^{0}(\mathcal{F})=H^{0}(\mathfrak{n})$.

- $H^{1}(\mathcal{F})=H^{1}(\mathfrak{n})$.

Hence we have the following.

COROLLARY 31. Let $N$ be a connected simply connected nilpotent Lie group, and let $M \stackrel{\rho_{0}}{n}$ $N$ be a $C^{\infty}$ locally free action. Then the following are equivalent.

- $\quad$ The action $\rho_{0}$ is parameter rigid.

- $H^{1}(\mathcal{F})=H^{1}(\mathfrak{n})$.

Proof. This is true even if $N=1$.

If we have vanishing of $H^{0}$ for the trivial coefficient, then we can deduce vanishing of $H^{0}$ for various non-trivial coefficients by an easy argument. This will be done in Lemma 40 in $\$ 7$.

The second necessary condition is on the vanishing of $H^{1}$. The following will be proved in $\S 8$.

THEOREM 32. (Vanishing of $H^{1}$ ) Let $V \subset \mathfrak{s}$ be an ad-invariant subspace (i.e. an ideal of s) for which $\mathfrak{n} \stackrel{\text { ad }}{\curvearrowright} V$ is trivial. Assume that any eigenvalue of ad $X$ on $\mathfrak{s} / V$ either is 0 or has non-zero real part for any $X \in \mathfrak{s}$. If $M \stackrel{\rho_{0}}{\curvearrowleft} S$ is parameter rigid, then we have

$$
H^{1}(\mathcal{F} ; \mathfrak{s} \stackrel{\text { ad }}{\curvearrowright} V)=H^{0}(\mathcal{F}) \otimes H^{1}(\mathfrak{s} ; \mathfrak{s} \stackrel{\text { ad }}{\curvearrowright} V)
$$

Note that the assumption is weaker than the assumption that $S$ is of exponential type, as it allows ad $X: V \rightarrow V$ to have purely imaginary non-zero eigenvalues.

Here an element $[\omega] \in H^{1}(\mathcal{F} ; \mathfrak{s} \stackrel{\text { ad }}{\curvearrowright} V)$ is in $H^{0}(\mathcal{F}) \otimes H^{1}(\mathfrak{s} ; \mathfrak{s} \stackrel{\text { ad }}{\curvearrowright} V)$ if and only if $[\omega]$ is represented by a leafwise constant form, that is, represented by a form $\phi \circ \omega_{0}$ for some $C^{\infty}$ leafwise constant map $\phi: M \rightarrow \operatorname{Hom}(\mathfrak{s}, V)$. If we assume also that $\mathfrak{s}$ has an outer derivation, then by Theorem 28, the conclusion simplifies to $H^{1}(\mathcal{F} ; \mathfrak{s} \stackrel{\text { ad }}{\curvearrowright} V)=H^{1}(\mathfrak{s} ; \mathfrak{s} \stackrel{\text { ad }}{\curvearrowright} V)$.

Let us consider the coefficients appearing Theorem 32. We have $V \subset \mathfrak{n}$; thus $V$ is contained in the center of $\mathfrak{n}$, and is an abelian ideal of $\mathfrak{s}$. (For the first part, if not, take $X \in V \backslash \mathfrak{n}$; then $\mathfrak{n}+\mathbb{R} X$ would be a nilpotent ideal of $\mathfrak{s}$ which is larger than the nilradical $\mathfrak{n}$.)

As an example of a coefficient $V$ satisfying the property, we can take $V=\mathfrak{n}^{s}$, where $\mathfrak{n} \supset \mathfrak{n}^{2} \supset \cdots \supset \mathfrak{n}^{s} \supset 0$ is the lower central series of $\mathfrak{n}$. 
As a more concrete example, we consider the two-dimensional solvable Lie algebra $\mathfrak{g a}=\mathbb{R} X \oplus \mathbb{R} Y$ defined by $[X, Y]=Y$. Then the one-dimensional representation $\mathfrak{g a} \stackrel{\text { ad }}{\curvearrowright}$ $\mathbb{R} Y$ satisfies the condition of Theorem 32 , but the trivial representation $\mathfrak{s} \curvearrowright \mathfrak{g a} / \mathbb{R} Y$ does not satisfy the condition.

\section{Vanishing of $H^{0}$-proof of Theorem 28}

The proof of Theorem 28 is immediate after proving Lemma 37, whose proof is the main part of this section. Several lemmas before Lemma 37 prepare an 'integration' map $\mu$, which will be used in the proof of Lemma 37. Sublemma 1 inside Lemma 37 is similar to Lemma 43 in the next section, and the same kind of argument already appeared in Maruhashi [16], where the vanishing of $H^{1}$ was proved under the assumption of parameter rigidity together with the vanishing of $H^{0}$ for actions of nilpotent Lie groups.

Let $M \stackrel{\rho_{0}}{\curvearrowleft} S$ be a $C^{\infty}$ locally free action of a connected simply connected solvable Lie group $S$ on a closed $C^{\infty}$ manifold $M$, with the orbit foliation $\mathcal{F}$ and the canonical 1 -form $\omega_{0}$.

Let $\mathfrak{s} \stackrel{\pi}{\curvearrowright} V$ be a finite-dimensional real representation, and let $S \stackrel{\Pi}{\curvearrowright} V$ denote the representation whose differentiation is $\pi$. Then the trivial bundle $M \times V \rightarrow M$ is an $S$-equivariant vector bundle with the action defined by

$$
(x, v) s=\left(\rho_{0}(x, s), \Pi\left(s^{-1}\right) v\right) .
$$

Let $\Gamma_{b l c}(V)$ be the space of all bounded sections of $M \times V \rightarrow M$ which are continuous on each leaf. (An element $\xi \in \Gamma_{b l c}(V)$ can be discontinuous on $M$.) We have a representation $S \curvearrowright \Gamma_{b l c}(V)$ by

$$
(s \xi)(x)=\Pi(s) \xi\left(\rho_{0}(x, s)\right)
$$

for $s \in S, \xi \in \Gamma_{b l c}(V)$ and $x \in M$. We equip $V$ with a norm coming from an inner product. Then $\Gamma_{b l c}(V)$ is a Banach space with the supremum norm. Let $\Gamma_{l c}(V)$ be the closed subspace of $\Gamma_{b l c}(V)$ which consists of bounded leafwise constant sections.

LEMMA 33. There is an S-equivariant continuous linear map

$$
\mu: \Gamma_{b l c}(V) \rightarrow \Gamma_{l c}(V)
$$

which is the identity on $\Gamma_{l c}(V)$.

Proof. Since $S$ is amenable, by one of the characterizations of amenability, we have a bi-invariant mean $\mu_{0}: C_{b}(S) \rightarrow \mathbb{R}$ on the space $C_{b}(S)$ of all bounded continuous real-valued functions on $S$. See Greenleaf [6, pp. 26-29]. Recall that $\mu_{0}(1)=1$ and its operator norm is 1 . Take a basis $v_{1}, \ldots, v_{n}$ of $V$. For $\xi=\sum_{i=1}^{n} f_{i} v_{i} \in \Gamma_{b l c}(V)$ and $x \in M$, we define

$$
\mu(\xi)(x)=\sum_{i=1}^{n} \mu_{0}\left(f_{i}\left(\rho_{0}(x, \cdot)\right)\right) v_{i}
$$


Then this is independent of the choice of a basis of $V$. We have

$$
\mu(\xi)\left(\rho_{0}(x, s)\right)=\sum_{i=1}^{n} \mu_{0}\left(f_{i}\left(\rho_{0}(x, s \cdot)\right)\right) v_{i}=\mu(\xi)(x)
$$

by left-invariance, and

$$
\mu(s \xi)(x)=\Pi(s) \sum_{i=1}^{n} \mu_{0}\left(f_{i}\left(\rho_{0}(x, \cdot s)\right)\right) v_{i}=s \mu(\xi)(x)
$$

by right-invariance. We also have $\mu(\xi)=\xi$ for $\xi \in \Gamma_{l c}(V)$ since $\mu_{0}(1)=1$. By taking $v_{1}, \ldots, v_{n}$ to be an orthonormal basis and using $\left\|\mu_{0}\right\|=1$, we see that

$$
\|\mu(\xi)(x)\|^{2} \leq \sum_{i=1}^{n}\left\|f_{i}\right\|_{\infty}^{2} \leq n\|\xi\|_{\infty}^{2} .
$$

Let $\nabla$ denote the flat leafwise connection of $M \times V \rightarrow M$ defined by $\stackrel{i}{\curvearrowright} V$.

LEMMA 34. For $v \in V, x_{0} \in M$ and sufficiently small $s \in S$, the locally defined section

$$
\xi_{0}\left(\rho_{0}\left(x_{0}, s\right)\right)=\left(\rho_{0}\left(x_{0}, s\right), \Pi\left(s^{-1}\right) v\right)
$$

of $M \times V \rightarrow M$ on the leaf containing $x_{0}$ is a parallel section for $\nabla$; that is, $\nabla \xi_{0}=0$.

Proof. For any $y=\rho_{0}\left(x_{0}, s_{0}\right)$ with small $s_{0} \in S$ and any $X \in \mathfrak{s}$, we have

$$
\begin{aligned}
\nabla_{\left.\frac{d}{d t} \rho_{0}\left(y, e^{t X}\right)\right|_{t=0} \xi_{0}} & =d_{\mathcal{F}} \xi_{0}\left(\left.\frac{d}{d t} \rho_{0}\left(y, e^{t X}\right)\right|_{t=0}\right)+\pi(X) \xi_{0}(y) \\
& =\left.\frac{d}{d t} \Pi\left(e^{-t X} s_{0}^{-1}\right) v\right|_{t=0}+\pi(X) \Pi\left(s_{0}^{-1}\right) v \\
& =0 .
\end{aligned}
$$

Therefore, the directions of orbits of the action $M \times V \curvearrowleft S$ are horizontal for the leafwise connection $\nabla$. By the expression for covariant derivative by parallel transport, we have

$$
\begin{aligned}
\left(\nabla_{X} \xi\right)(x) & =\lim _{t \rightarrow 0} \frac{\Pi\left(e^{t X}\right) \xi\left(\rho_{0}\left(x, e^{t X}\right)\right)-\xi(x)}{t} \\
& =\lim _{t \rightarrow 0} \frac{\left(e^{t X} \xi\right)(x)-\xi(x)}{t}
\end{aligned}
$$

for any $\xi \in \Gamma(V), X \in \mathfrak{s}$ and $x \in M$. Note that $X \in \mathfrak{s}$ is regarded as $X \in \Gamma(T \mathcal{F})$ using the locally free action $\rho_{0}$.

LEMma 35. For any $\xi \in \Gamma(V)$ and $X \in \mathfrak{s},\left(e^{t X} \xi-\xi\right) / t$ converges uniformly to $\nabla_{X} \xi$ as $t \rightarrow 0$.

Proof. Take a basis $v_{1}, \ldots, v_{n}$ of $V$ and write $\left(e^{t X} \xi\right)(x)=\sum_{i=1}^{n} f_{i}(t, x) v_{i}$ for some $C^{\infty}$ functions $f_{i}: \mathbb{R} \times M \rightarrow \mathbb{R}$. Then we have $\left(\nabla_{X} \xi\right)(x)=\sum_{i=1}^{n} f_{i}^{\prime}(0, x) v_{i}$. The function 
$f_{i}(t, x)$ has the Taylor expansion

$$
f_{i}(t, x)=f_{i}(0, x)+t f_{i}^{\prime}(0, x)+\frac{t^{2}}{2} f_{i}^{\prime \prime}\left(\theta_{i, x, t}, x\right),
$$

where $\theta_{i, x, t}$ is a number between 0 and $t$. Since

$$
\frac{\left(e^{t X} \xi\right)(x)-\xi(x)}{t}-\left(\nabla_{X} \xi\right)(x)=\frac{t}{2} \sum_{i=1}^{n} f_{i}^{\prime \prime}\left(\theta_{i, x, t}, x\right) v_{i}
$$

and $f_{i}^{\prime \prime}(\theta, x)$ is bounded for $-1 \leq \theta \leq 1$ and $x \in M$, we get the conclusion.

Lemma 36. Let $\mu: \Gamma_{b l c}(V) \rightarrow \Gamma_{l c}(V)$ be the map in Lemma 33. Then

$$
\mu\left(\nabla_{X} \xi\right)=\nabla_{X} \mu(\xi)
$$

for all $\xi \in \Gamma(V)$ and $X \in \mathfrak{s}$. (Note that $\mu(\xi)$ might be discontinuous on $M$.)

Proof. By Lemma 35, $\left(e^{t X} \xi-\xi\right) / t$ converges uniformly to $\nabla_{X} \xi$ as $t \rightarrow 0$. By continuity and equivariance, we have

$$
\mu\left(\nabla_{X} \xi\right)=\lim _{t \rightarrow 0} \frac{e^{t X} \mu(\xi)-\mu(\xi)}{t}=\nabla_{X} \mu(\xi) .
$$

Lemma 37. Assume that $S$ is of exponential type. Let $\Psi: M \rightarrow \operatorname{Aut}(S)$ be a $C^{\infty}$ map which is constant on each leaf of $\mathcal{F}$. If $\rho_{0}$ is parameter rigid, then $\bar{\Psi}: M \rightarrow \operatorname{Out}(S)$ is constant on $M$, where the bar denotes the projection $\operatorname{Aut}(S) \rightarrow \operatorname{Out}(S)$. In particular, if $\operatorname{Out}(S) \neq 1, M$ must be connected.

Proof. Define $M \stackrel{\rho}{\curvearrowleft} S$ by $\rho(x, s)=\rho_{0}\left(x, \Psi_{x}^{-1}(s)\right)$. This defines an action because $\Psi$ is leafwise constant:

$$
\begin{aligned}
\rho\left(x, s s^{\prime}\right) & =\rho_{0}\left(\rho_{0}\left(x, \Psi_{x}^{-1}(s)\right), \Psi_{x}^{-1}\left(s^{\prime}\right)\right) \\
& =\rho_{0}\left(\rho(x, s), \Psi_{\rho(x, s)}^{-1}\left(s^{\prime}\right)\right) \\
& =\rho\left(\rho(x, s), s^{\prime}\right) .
\end{aligned}
$$

Since $\rho$ is a $C^{\infty}$ locally free action with the same orbit foliation as $\rho_{0}, \rho$ is parameter equivalent to $\rho_{0}$ by parameter rigidity. Note that $\Psi_{x *} \omega_{0}$ is the canonical 1 -form of $\rho$. By Proposition 1.4.4 of Asaoka [2], there exist $\Phi \in \operatorname{Aut}(S)$ and a $C^{\infty}$ map $P: M \rightarrow S$ such that

$$
\Psi_{x *} \omega_{0}=\operatorname{Ad}\left(P^{-1}\right) \Phi_{*} \omega_{0}+P^{*} \Theta,
$$

where $\Theta$ denotes the left Maurer-Cartan form of $S$. (In [2], $\Phi$ is referred to as an endomorphism, but it is the same $\Phi$ that appears in the definition of parameter equivalence which we saw in $\S 2$, so $\Phi$ can be taken as an automorphism. It is easy to see $P^{*} \Theta$ is equivalent to the expression $P^{-1} d_{\mathcal{F}} P$ in [2]. There is a small difference between our definition of parameter equivalence and the one in [2], since in [2] the map $F$ is assumed to be homotopic to the identity through diffeomorphisms. But this does not cause any problem here.) 
Let $a$ denote both projections $\mathfrak{s} \rightarrow \mathfrak{s} / \mathfrak{n}$ and $S \rightarrow S / N$, where $\mathfrak{n}$ is the nilradical of $\mathfrak{s}$ and $N$ is the Lie subgroup corresponding to $\mathfrak{n}$. By projecting (13), we get

$$
a \Psi_{x *} \omega_{0}=a \Phi_{*} \omega_{0}+d_{\mathcal{F}} a P,
$$

since $\mathfrak{s} / \mathfrak{n}$ is abelian. For any $x \in M, X \in \mathfrak{s}$ and $T>0$, we integrate (14) over the curve $\rho_{0}\left(x, e^{t X}\right)$ for $0 \leq t \leq T$. Then, noting that $\Psi$ is leafwise constant, we have

$$
\operatorname{Ta} \Psi_{x *} X=\operatorname{Ta} \Phi_{*} X+a P\left(\rho_{0}\left(x, e^{T X}\right)\right)-a P(x) .
$$

Since $a P$ is bounded due to the compactness of $M$, we must have $a \Psi_{x *} X=a \Phi_{*} X$, and $a P$ is leafwise constant. Hence there exists a leafwise constant $C^{\infty}$ map $R: M \rightarrow S$ such that $Q=R^{-1} P: M \rightarrow N$. Since $R$ is leafwise constant, we have

$$
P^{*} \Theta=(R Q)^{*} \Theta=Q^{*} \Theta
$$

and (13) becomes

$$
\Psi_{x *} \omega_{0}=\operatorname{Ad}\left(Q^{-1}\right) \operatorname{Ad}\left(R^{-1}\right) \Phi_{*} \omega_{0}+Q^{*} \Theta .
$$

Let $\mathfrak{n} \supset \mathfrak{n}^{2} \supset \cdots \supset \mathfrak{n}^{s} \supset 0$ be the lower central series of $\mathfrak{n}$. Recall that exp: $\mathfrak{n} \rightarrow N$ is a diffeomorphism and $\log : N \rightarrow \mathfrak{n}$ is defined.

Sublemma 38. Assume that there exist a $C^{\infty}$ map $Q: M \rightarrow N$ and a leafwise constant $C^{\infty}$ map $R: M \rightarrow S$ such that:

- $\Psi_{x *} \omega_{0}=\operatorname{Ad}\left(Q^{-1}\right) \operatorname{Ad}\left(R^{-1}\right) \Phi_{*} \omega_{0}+Q^{*} \Theta$;

- $\log Q \in \mathfrak{n}^{k}$ for some $1 \leq k \leq s$.

Then we can find a $C^{\infty}$ map $Q^{\prime}: M \rightarrow N$ and a leafwise constant $C^{\infty}$ map $R^{\prime}: M \rightarrow S$ such that:

- $\Psi_{x *} \omega_{0}=\operatorname{Ad}\left(\left(Q^{\prime}\right)^{-1}\right) \operatorname{Ad}\left(\left(R^{\prime}\right)^{-1}\right) \Phi_{*} \omega_{0}+\left(Q^{\prime}\right)^{*} \Theta$

- $\log Q^{\prime} \in \mathfrak{n}^{k+1}$.

Proof. Take subspaces $V_{0}, \ldots, V_{s}$ such that $\mathfrak{s}=V_{0} \oplus \mathfrak{n}$ and $\mathfrak{n}^{i}=V_{i} \oplus \mathfrak{n}^{i+1}$ for $i=$ $1, \ldots, s$. We can write $Q=\exp \left(\sum_{i=k}^{s} Q_{i}\right)$ for some $C^{\infty}$ maps $Q_{i}: M \rightarrow V_{i}$. We will calculate the $V_{k}$ component of

$$
\Psi_{x *} \omega_{0}=\operatorname{Ad}\left(Q^{-1}\right) \operatorname{Ad}\left(R^{-1}\right) \Phi_{*} \omega_{0}+Q^{*} \Theta .
$$

First note that

$$
Q^{*} \Theta \equiv d_{\mathcal{F}} Q_{k} \bmod \mathfrak{n}^{k+1} \text {. }
$$

In fact, for all $X=\left.\frac{d}{d t} x(t)\right|_{t=0} \in T_{x} \mathcal{F}$,

$$
\begin{aligned}
Q^{*} \Theta(X) & =\left.\frac{d}{d t} Q(x)^{-1} Q(x(t))\right|_{t=0} \\
& =\left.\frac{d}{d t} \exp \left(-\sum_{i=k}^{s} Q_{i}(x)\right) \exp \left(\sum_{i=k}^{s} Q_{i}(x(t))\right)\right|_{t=0} \\
& =\left.\frac{d}{d t} \exp \left(\sum_{i=k}^{s}\left(Q_{i}(x(t))-Q_{i}(x)\right)+\text { an element of } \mathfrak{n}^{k+1}\right)\right|_{t=0}
\end{aligned}
$$




$$
\begin{aligned}
& =\left.\frac{d}{d t} \exp \left(Q_{k}(x(t))-Q_{k}(x)+\text { an element of } \mathfrak{n}^{k+1}\right)\right|_{t=0} \\
& \equiv d_{\mathcal{F}} Q_{k}(X) \bmod \mathfrak{n}^{k+1}
\end{aligned}
$$

Let $\mathfrak{s} \stackrel{\pi_{k}^{0}}{\curvearrowright} V_{k}$ be the representation obtained from $\mathfrak{s} \stackrel{\text { ad }}{\curvearrowright} \mathfrak{n}^{k} / \mathfrak{n}^{k+1}$ by the identification $V_{k} \simeq$

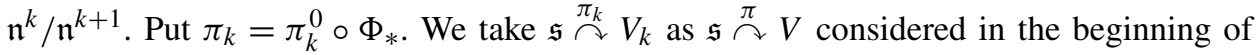
this section; we let $\nabla$ be the leafwise connection defined by $\pi_{k}$, and we let $\mu: \Gamma_{b l c}\left(V_{k}\right) \rightarrow$ $\Gamma_{l c}\left(V_{k}\right)$ be the map in Lemma 33.

Write $\Psi_{x *} \omega_{0}=\sum_{i=0}^{s} \alpha_{i}$ and $\operatorname{Ad}\left(R^{-1}\right) \Phi_{*} \omega_{0}=\sum_{i=0}^{s} \beta_{i}$ according to the decomposition $\mathfrak{s}=\bigoplus_{i=0}^{s} V_{i}$. Then we have

$$
\begin{aligned}
\operatorname{Ad}\left(Q^{-1}\right) \operatorname{Ad}\left(R^{-1}\right) \Phi_{*} \omega_{0} & =\exp \left(\operatorname{ad}\left(-\sum_{i=k}^{s} Q_{i}\right)\right) \sum_{i=0}^{s} \beta_{i} \\
& \equiv \sum_{i=0}^{k} \beta_{i}+\left[\beta_{0}, Q_{k}\right] \bmod \mathfrak{n}^{k+1} \\
& \equiv \sum_{i=0}^{k} \beta_{i}+\pi_{k}^{0} \beta_{0} Q_{k} \bmod \mathfrak{n}^{k+1}
\end{aligned}
$$

Take the $V_{k}$ components of (16) to get

$$
\alpha_{k}=\beta_{k}+\pi_{k}^{0} \beta_{0} Q_{k}+d_{\mathcal{F}} Q_{k} .
$$

Since

$$
\Phi_{*} \omega_{0} \equiv \operatorname{Ad}\left(R^{-1}\right) \Phi_{*} \omega_{0} \equiv \beta_{0} \bmod \mathfrak{n}
$$

and $\pi_{k}^{0}$ vanishes on $\mathfrak{n}$, we have $\pi_{k} \omega_{0}=\pi_{k}^{0} \beta_{0}$. Therefore,

$$
\begin{aligned}
\nabla Q_{k} & =d_{\mathcal{F}} Q_{k}+\pi_{k} \omega_{0} Q_{k} \\
& =d_{\mathcal{F}} Q_{k}+\pi_{k}^{0} \beta_{0} Q_{k},
\end{aligned}
$$

and we get

$$
\alpha_{k}=\beta_{k}+\nabla Q_{k}
$$

Hence

$$
\alpha_{k}(X)=\beta_{k}(X)+\nabla_{X} Q_{k}
$$

for any $X \in \mathfrak{s}$. Note that $\alpha_{k}(X)$ and $\beta_{k}(X)$ are leafwise constant because $R$ is leafwise constant. Applying $\mu$ and using Lemma 36, we get

$$
\alpha_{k}(X)=\beta_{k}(X)+\nabla_{X} \mu\left(Q_{k}\right) .
$$

Therefore,

$$
\nabla_{X}\left(Q_{k}-\mu\left(Q_{k}\right)\right)=0 .
$$


Put $Q_{k}^{\prime}=Q_{k}-\mu\left(Q_{k}\right)$. We shall see $Q_{k}^{\prime}$ is leafwise constant. Let $S \stackrel{\Pi_{k}}{\curvearrowright} V_{k}$ be the representation with the derivative $\mathfrak{s} \stackrel{\pi_{k}}{\Omega} V_{k}$. Then for any $t \in \mathbb{R}$ and $x \in M$, we have

$$
\begin{aligned}
\left.\frac{d}{d s}\right|_{s=t}\left(e^{s X} Q_{k}^{\prime}\right)(x) & =\lim _{h \rightarrow 0} \Pi_{k}\left(e^{t X}\right) \frac{\left(e^{h X} Q_{k}^{\prime}\right)\left(\rho_{0}\left(x, e^{t X}\right)\right)-Q_{k}^{\prime}\left(\rho_{0}\left(x, e^{t X}\right)\right)}{h} \\
& =\Pi_{k}\left(e^{t X}\right)\left(\nabla_{X} Q_{k}^{\prime}\right)\left(\rho_{0}\left(x, e^{t X}\right)\right) \\
& =0 .
\end{aligned}
$$

Thus $\left(e^{t X} Q_{k}^{\prime}\right)(x)=\Pi_{k}\left(e^{t X}\right) Q_{k}^{\prime}\left(\rho_{0}\left(x, e^{t X}\right)\right)$ is constant with respect to $t$. So

$$
Q_{k}^{\prime}\left(\rho_{0}\left(x, e^{t X}\right)\right)=\Pi_{k}\left(e^{-t X}\right) Q_{k}^{\prime}(x)=e^{-t \pi_{k}(X)} Q_{k}^{\prime}(x)
$$

for all $t \in \mathbb{R}$. Note that $Q_{k}^{\prime}\left(\rho_{0}\left(x, e^{t X}\right)\right)$ is bounded with respect to $t$. Take a basis of $V_{k}$ which turns $-\pi_{k}(X)=-\pi_{k}^{0}\left(\Phi_{*} X\right)$ into a real Jordan normal form. Since any eigenvalue of ad $X: \mathfrak{s} \rightarrow \mathfrak{s}$ for any $X \in \mathfrak{s}$ either is 0 or has non-zero real part by our assumption that $\mathfrak{s}$ is of exponential type, the same is true for $\pi_{k}^{0}(X): V_{k} \rightarrow V_{k}$ for all $X \in \mathfrak{s}$. Therefore, each Jordan block of $-\pi_{k}(X)=-\pi_{k}^{0}\left(\Phi_{*} X\right)$ has eigenvalue either equal to 0 or with non-zero real part. For a Jordan block whose eigenvalue has non-zero real part, the corresponding components of $e^{-t \pi_{k}(X)} Q_{k}^{\prime}(x)$ have the following forms:

$$
\left(\begin{array}{ccc}
e^{t a} & & * \\
& \ddots & \\
0 & & e^{t a}
\end{array}\right)\left(\begin{array}{c}
c_{1} \\
\vdots \\
c_{m}
\end{array}\right)
$$

if the eigenvalue $a$ is real, and

$$
\left(\begin{array}{ccc}
e^{t a} R_{t} & & * \\
& \ddots & \\
0 & & e^{t a} R_{t}
\end{array}\right)\left(\begin{array}{c}
c_{1} \\
\vdots \\
c_{m}
\end{array}\right),
$$

where

$$
R_{t}=\left(\begin{array}{cc}
\cos t b & \sin t b \\
-\sin t b & \cos t b
\end{array}\right)
$$

if the eigenvalue $a+b i$ is not real. Since this must be bounded for all $t \in \mathbb{R}, c_{1}=\cdots=$ $c_{m}=0$, which implies the corresponding components of $Q_{k}^{\prime}\left(\rho_{0}\left(x, e^{t X}\right)\right)$ must be constant.

On the other hand, for a Jordan block with eigenvalue 0 , the corresponding components in $e^{-t \pi_{k}(X)} Q_{k}^{\prime}(x)$ is

$$
\left(\begin{array}{ccc}
1 & & * \\
& \ddots & \\
0 & & 1
\end{array}\right)\left(\begin{array}{c}
c_{1} \\
\vdots \\
c_{m}
\end{array}\right),
$$

where the entries in the $*$ part of the matrix are now polynomials in $t$. Since bounded polynomial functions must be constant, we see that the corresponding components in $Q_{k}^{\prime}\left(\rho_{0}\left(x, e^{t X}\right)\right)$ are also constant. 
So $Q_{k}$ is leafwise constant. Put $Q^{\prime}=e^{-Q_{k}} Q$. Then $\log Q^{\prime}$ has values in $\mathfrak{n}^{k+1}$ and

$$
\Psi_{x *} \omega_{0}=\operatorname{Ad}\left(\left(Q^{\prime}\right)^{-1}\right) \operatorname{Ad}\left(\left(R^{\prime}\right)^{-1}\right) \Phi_{*} \omega_{0}+\left(Q^{\prime}\right)^{*} \Theta,
$$

where $R^{\prime}=R e^{Q_{k}}$ is leafwise constant.

Applying Sublemma 1 to (15) repeatedly, we finally get $Q=1$ and therefore

$$
\Psi_{x *} \omega_{0}=\operatorname{Ad}\left(R^{-1}\right) \Phi_{*} \omega_{0}
$$

for some $R$. Therefore, $\Psi_{x}$ is equal to $\Phi$ modulo inner automorphisms.

Theorem 28 is restated and proved here.

THEOREM 39. Assume that $S$ is of exponential type and there is an outer derivation of $\mathfrak{s}$. If $M \stackrel{\rho_{0}}{\curvearrowleft} S$ is parameter rigid, then $M$ is connected and $H^{0}(\mathcal{F})=H^{0}(\mathfrak{s})$.

Proof. Since there is an outer derivation of $\mathfrak{s}$, the outer automorphism group $\operatorname{Out}(S)$ of $S$ is non-trivial; hence $M$ is connected. Take an outer derivation $\varphi$ of $\mathfrak{s}$ and set $\Phi_{t}=e^{t \varphi} \in$ $\operatorname{Aut}(S)$. For any $f \in H^{0}(\mathcal{F})$, consider a map $M \rightarrow \operatorname{Aut}(S)$ defined by $x \mapsto \Phi_{f(x)}$. Since this is leafwise constant, $x \mapsto \overline{\Phi_{f(x)}} \in \operatorname{Out}(S)$ is constant by Lemma 37. Let $\operatorname{Inn}(S)$ denote the inner automorphism group of $S$. This is a connected normal Lie subgroup of $\operatorname{Aut}(S)$. We must be a bit careful because $\operatorname{Inn}(S)$ might not be closed in $\operatorname{Aut}(S)$ in general. See Hochschild [8]. But the cosets of $\operatorname{Inn}(S)$ define a foliation on $\operatorname{Aut}(S)$, and $\Phi_{t}$ is a curve transverse to the foliation. Since the automorphisms $\Phi_{f(x)}$ for all $x \in M$ are contained in a single leaf of $\mathcal{F}$ and $M$ is connected, $\Phi_{f(x)}$ must be constant with respect to $x$. This implies $f$ is constant over $M$.

Finally we see the vanishing of $H^{0}$ with non-trivial coefficients.

LEMMA 40. Assume $H^{0}(\mathcal{F})=H^{0}(\mathfrak{s})$. Let $\mathfrak{s} \stackrel{\pi}{\curvearrowright} V$ be a representation for which $\pi(X)$ has no non-zero purely imaginary eigenvalues for each $X \in \mathfrak{s}$. Then $H^{0}(\mathcal{F} ; \pi)=H^{0}(\mathfrak{s} ; \pi)$.

Proof. Take $\xi \in H^{0}(\mathcal{F} ; \pi)$. The function $\xi$ satisfies $d_{\mathcal{F}} \xi+\pi \omega_{0} \xi=0$. This means $X \xi+$ $\pi(X) \xi=0$ for all $X \in \mathfrak{s}$. For each $x \in M$ this is solved as $\xi\left(\rho_{0}\left(x, e^{t X}\right)\right)=e^{-t \pi(X)} \xi(x)$ for all $t \in \mathbb{R}$. As in the proof of Sublemma 1 we transform $\pi(X)$ into a real Jordan normal form, and $\xi$ being bounded implies $\xi\left(\rho_{0}\left(x, e^{t X}\right)\right)$ must be constant. Therefore, $\xi$ is leafwise constant. By the assumption $H^{0}(\mathcal{F})=H^{0}(\mathfrak{s}), \xi$ is constant on $M$. Hence $\xi \in V$ and $\pi(X) \xi=0$ for all $X \in \mathfrak{s}$, which shows $\xi \in H^{0}(\mathfrak{s} ; \pi)$.

\section{Vanishing of $H^{1}$ - proof of Theorem 32}

Here we prove the following (a restatement of Theorem 32).

THEOREM 41. Let $V \subset \mathfrak{s}$ be an ad-invariant subspace (i.e. an ideal of $\mathfrak{s}$ ) for which $\mathfrak{n} \stackrel{\text { ad }}{\curvearrowright} V$ is trivial. Assume that any eigenvalue of ad $X$ on $\mathfrak{s} / V$ either is 0 or has non-zero real part for any $X \in \mathfrak{s}$. If $M \stackrel{\rho_{0}}{\curvearrowleft} S$ is parameter rigid, then we have

$$
H^{1}(\mathcal{F} ; \mathfrak{s} \stackrel{\text { ad }}{\curvearrowright} V)=H^{0}(\mathcal{F}) \otimes H^{1}(\mathfrak{s} ; \mathfrak{s} \stackrel{\text { ad }}{\curvearrowright} V)
$$


Proof. Take any $[\omega] \in H^{1}(\mathcal{F} ; \mathfrak{s} \stackrel{\text { ad }}{\curvearrowright} V)$. Let $\omega_{0}$ be the canonical 1-form of $\rho_{0}$. Fix an $\epsilon>0$ and put $\eta:=\omega_{0}+\epsilon \omega \in \Gamma(\operatorname{Hom}(T \mathcal{F}, \mathfrak{s}))$. Let us see that $\eta$ satisfies the Maurer-Cartan equation. As we saw in $\S 6, V$ is abelian, and then

$$
\begin{aligned}
d_{\mathcal{F}} \eta+[\eta, \eta] & =d_{\mathcal{F}} \omega_{0}+\epsilon d_{\mathcal{F}} \omega+\left[\omega_{0}, \omega_{0}\right]+\epsilon\left(\left[\omega_{0}, \omega\right]+\left[\omega, \omega_{0}\right]\right) \\
& =\epsilon\left(d_{\mathcal{F}} \omega+\left[\omega_{0}, \omega\right]+\left[\omega, \omega_{0}\right]\right) .
\end{aligned}
$$

But this is zero, because $\omega$ satisfies $d_{\mathcal{F}} \omega+\left(\operatorname{ad} \omega_{0}\right) \wedge \omega=0$ and $\left(\operatorname{ad} \omega_{0}\right) \wedge \omega=\left[\omega_{0}, \omega\right]+$ $\left[\omega, \omega_{0}\right]$.

Since $M$ is compact, we can assume $\eta_{x}: T_{x} \mathcal{F} \rightarrow \mathfrak{s}$ is bijective for all $x \in M$ by taking $\epsilon>0$ small enough. Then there exists a unique action $\rho$ of $S$ on $M$ whose orbit foliation is $\mathcal{F}$ and whose canonical 1 -form is $\eta$. See Asaoka [2, Proposition 1.4.3]. By parameter rigidity, $\rho$ is parameter equivalent to $\rho_{0}$. Thus by [2, Proposition 1.4.4], there exist a $C^{\infty}$ map $P: M \rightarrow S$ and an automorphism $\Phi$ of $S$ satisfying

$$
\omega_{0}+\epsilon \omega=\operatorname{Ad}\left(P^{-1}\right) \Phi_{*} \omega_{0}+P^{*} \Theta,
$$

where $\Theta$ is the left Maurer-Cartan form of $S$. By considering this equation modulo $\mathfrak{n}$, we get

$$
\omega_{0} \equiv \Phi_{*} \omega_{0}+d_{\mathcal{F}} \bar{P} \bmod \mathfrak{n},
$$

where the bar denotes the projection $S \rightarrow S / N$. The same argument as in the proof of vanishing of $H^{0}$ yields

$$
\begin{aligned}
\omega_{0} & \equiv \Phi_{*} \omega_{0} \bmod \mathfrak{n}, \\
d_{\mathcal{F}} \bar{P} & \equiv 0 \bmod \mathfrak{n} .
\end{aligned}
$$

So we can take a leafwise constant $C^{\infty}$ map $R: M \rightarrow S$ such that $Q:=R^{-1} P \in N$. Then equation (18) becomes

$$
\begin{aligned}
\omega_{0}+\epsilon \omega & =\operatorname{Ad}\left(Q^{-1} R^{-1}\right) \Phi_{*} \omega_{0}+(R Q)^{*} \Theta \\
& =\operatorname{Ad}\left(Q^{-1}\right) \Psi_{*} \omega_{0}+Q^{*} \Theta,
\end{aligned}
$$

where $\Psi_{*}=\operatorname{Ad}\left(R^{-1}\right) \Phi_{*}$ is leafwise constant.

LEMMA 42. There exists a filtration

$$
\mathfrak{s} \supset \mathfrak{n}=W_{1} \supset W_{2} \supset \cdots \supset W_{s}=V \supset W_{s+1}=0,
$$

where the $W_{i}$ are ideals of $\mathfrak{s}$ such that $\left[\mathfrak{n}, W_{i}\right] \subset W_{i+1}$.

Proof. If

$$
\mathfrak{n} \supset \mathfrak{n}^{2} \supset \cdots \supset \mathfrak{n}^{s-1} \supset 0
$$

denotes the lower central series of $\mathfrak{n}$, then the filtration

$$
\mathfrak{s} \supset \mathfrak{n} \supset \mathfrak{n}^{2}+V \supset \mathfrak{n}^{3}+V \supset \cdots \supset \mathfrak{n}^{s-1}+V \supset V \supset 0
$$

gives the desired filtration.

Note that we have $\omega_{0} \equiv \Psi_{*} \omega_{0}$ modulo $W_{1}$. 
LEMmA 43. Assume there exist a $C^{\infty}$ map $Q: M \rightarrow N$ and a leafwise constant $C^{\infty}$ map $\Psi: M \rightarrow \operatorname{Aut}(S)$ such that

$$
\begin{aligned}
\omega_{0}+\epsilon \omega= & \operatorname{Ad}\left(Q^{-1}\right) \Psi_{*} \omega_{0}+Q^{*} \Theta, \\
& \log Q \in W_{k}
\end{aligned}
$$

and

$$
\omega_{0} \equiv \Psi_{*} \omega_{0} \bmod W_{k} .
$$

(1) If $k<s$, then there exist a $C^{\infty}$ map $Q^{\prime}: M \rightarrow N$ and a leafwise constant $C^{\infty}$ map $\Psi^{\prime}: M \rightarrow \operatorname{Aut}(S)$ such that

$$
\begin{aligned}
\omega_{0}+\epsilon \omega= & \operatorname{Ad}\left(\left(Q^{\prime}\right)^{-1}\right) \Psi_{*}^{\prime} \omega_{0}+\left(Q^{\prime}\right)^{*} \Theta, \\
& \log Q^{\prime} \in W_{k+1}
\end{aligned}
$$

and

$$
\omega_{0} \equiv \Psi_{*}^{\prime} \omega_{0} \bmod W_{k+1} .
$$

(2) If $k=s$, then $\omega$ is cohomologous to a leafwise constant cocycle.

Proof. The proof is similar to the proof of Sublemma 1. Take complementary subspaces $V_{i}$ so that $\mathfrak{s}=V_{0} \oplus \mathfrak{n}$ and $W_{i}=V_{i} \oplus W_{i+1}$. Write

$$
\omega_{0}=\sum_{i=0}^{s} \alpha_{i}, \quad \Psi_{*} \omega_{0}=\sum_{i=0}^{s} \beta_{i} \quad \text { and } \quad Q=\exp \left(\sum_{i=k}^{s} Q_{i}\right)
$$

according to the decomposition $\mathfrak{s}=\bigoplus_{i=0}^{s} V_{i}$.

The same calculation as in Sublemma 1 gives

$$
Q^{*} \Theta \equiv d_{\mathcal{F}} Q_{k} \quad \bmod W_{k+1} \text {. }
$$

We have

$$
\begin{aligned}
\operatorname{Ad}\left(Q^{-1}\right) \Psi_{*} \omega_{0} & \left.=\exp \left(\operatorname{ad}\left(-\sum_{i=k}^{s} Q_{i}\right)\right)\right) \sum_{i=0}^{s} \beta_{i} \\
& \equiv \sum_{i=0}^{k} \beta_{i}+\left[\beta_{0}, Q_{k}\right] \bmod W_{k+1} \\
& =\sum_{i=0}^{k-1} \alpha_{i}+\beta_{k}+\left[\alpha_{0}, Q_{k}\right] \bmod W_{k+1}
\end{aligned}
$$

Equation (19) gives

$$
\sum_{i=0}^{k} \alpha_{i}+\delta_{k s} \epsilon \omega \equiv \sum_{i=0}^{k-1} \alpha_{i}+\beta_{k}+\left[\alpha_{0}, Q_{k}\right]+d_{\mathcal{F}} Q_{k} \bmod W_{k+1} .
$$

Thus

$$
\alpha_{k}+\delta_{k s} \epsilon \omega \equiv \beta_{k}+\left[\alpha_{0}, Q_{k}\right]+d_{\mathcal{F}} Q_{k} \quad \bmod W_{k+1} .
$$

If $k=s$, we have

$$
\omega=\epsilon^{-1}\left(\beta_{S}-\alpha_{s}\right)+d_{\mathcal{F}}\left(\epsilon^{-1} Q_{s}\right)+\left[\alpha_{0}, \epsilon^{-1} Q_{s}\right] .
$$


If $\nabla$ denotes the covariant derivative defined from $\mathfrak{s} \stackrel{\text { ad }}{\curvearrowright} V$, then by $[\mathfrak{n}, V]=0$ we have

$$
\begin{aligned}
\nabla\left(\epsilon^{-1} Q_{s}\right) & =d_{\mathcal{F}}\left(\epsilon^{-1} Q_{s}\right)+\left[\omega_{0}, \epsilon^{-1} Q_{s}\right] \\
& =d_{\mathcal{F}}\left(\epsilon^{-1} Q_{s}\right)+\left[\alpha_{0}, \epsilon^{-1} Q_{s}\right]
\end{aligned}
$$

Therefore, $\omega$ is cohomologous to $\epsilon^{-1}\left(\beta_{s}-\alpha_{s}\right)$, which is leafwise constant since so are $\omega_{0}$ and $\Psi_{*} \omega_{0}$.

If $k<s$, then

$$
\alpha_{k} \equiv \beta_{k}+\left[\alpha_{0}, Q_{k}\right]+d_{\mathcal{F}} Q_{k} \bmod W_{k+1} .
$$

Let $\mathfrak{s} \stackrel{\pi_{k}}{\curvearrowright} V_{k}$ denote the representation obtained from $\mathfrak{s} \stackrel{\text { ad }}{\curvearrowright} W_{k} / W_{k+1}$ by the identification $W_{k} / W_{k+1} \simeq V_{k}$, and let $\nabla$ be the leafwise connection defined by $\pi_{k}$. Recall that $\nabla Q_{k}=$ $d_{\mathcal{F}} Q_{k}+\pi_{k} \omega_{0} Q_{k}$. Since

$$
\begin{aligned}
\pi_{k} \omega_{0} Q_{k} & =\pi_{k}\left(\sum_{i=0}^{s} \alpha_{i}\right) Q_{k} \\
& \equiv\left[\alpha_{0}, Q_{k}\right] \bmod W_{k+1},
\end{aligned}
$$

we have

$$
\alpha_{k} \equiv \beta_{k}+d_{\mathcal{F}} Q_{k}+\pi_{k} \omega_{0} Q_{k} \quad \bmod W_{k+1},
$$

which implies

$$
\begin{aligned}
\alpha_{k} & =\beta_{k}+d_{\mathcal{F}} Q_{k}+\pi_{k} \omega_{0} Q_{k} \\
& =\beta_{k}+\nabla Q_{k} .
\end{aligned}
$$

By the same argument as that starting from equation (17) in the proof of vanishing of $H^{0}$, using the assumption on the eigenvalues of ad $X$, we can conclude that $Q_{k}$ is leafwise constant. Define $Q^{\prime}: M \rightarrow N$ by $Q=e^{Q_{k}} Q^{\prime}$. Then equation (19) becomes

$$
\begin{aligned}
\omega_{0}+\epsilon \omega & =\operatorname{Ad}\left(\left(Q^{\prime}\right)^{-1} e^{-Q_{k}}\right) \Psi_{*} \omega_{0}+\left(e^{Q_{k}} Q^{\prime}\right)^{*} \Theta \\
& =\operatorname{Ad}\left(\left(Q^{\prime}\right)^{-1}\right) \Psi_{*}^{\prime} \omega_{0}+\left(Q^{\prime}\right)^{*} \Theta,
\end{aligned}
$$

where $\Psi_{*}^{\prime}=\operatorname{Ad}\left(e^{-Q_{k}}\right) \Psi_{*}$. Now we have $\log Q^{\prime} \in W_{k+1}$ and

$$
\begin{aligned}
\Psi_{*}^{\prime} \omega_{0} & =e^{- \text {ad } Q_{k}} \Psi_{*} \omega_{0} \\
& =e^{-\operatorname{ad} Q_{k}}\left(\sum_{i=0}^{k-1} \alpha_{i}+\beta_{k}+\text { an element of } W_{k+1}\right) \\
& \equiv \sum_{i=0}^{k-1} \alpha_{i}+\beta_{k}+\left[\alpha_{0}, Q_{k}\right] \quad \bmod W_{k+1} \\
& \equiv \sum_{i=0}^{k} \alpha_{i} \bmod W_{k+1} \\
& =\omega_{0} \bmod W_{k+1}
\end{aligned}
$$

since $d_{\mathcal{F}} Q_{k}=0$. 
Applying Lemma 43 repeatedly, we see that $\omega$ is cohomologous to a leafwise constant cocycle. Note that we have used the assumption on the eigenvalues only on $V_{1}, \ldots, V_{s-1}$, but not on $V_{s}=V$.

Acknowledgements. This paper was written during the author's stays at the Institut des Hautes Études Scientifiques in Bures-sur-Yvette and the Max-Planck-Institut für Mathematik in Bonn. The stays were supported by the Researcher Exchange Program between these institutes and the Japan Society for the Promotion of Science.

\section{REFERENCES}

[1] M. Asaoka. Nonhomogeneous locally free actions of the affine group. Ann. Math. (2) 175(1) (2012), 1-21.

[2] M. Asaoka. Deformation of locally free actions and leafwise cohomology. Foliations: Dynamics, Geometry and Topology (Advanced Courses in Mathematics - CRM Barcelona). Birkhäuser/Springer, Basel, $\mathrm{CH}$, 2014, pp. 1-40.

[3] E. Breuillard. Geometry of locally compact groups of polynomial growth and shape of large balls. Groups Geom. Dyn. 8(3) (2014), 669-732.

[4] J. Dixmier. L'application exponentielle dans les groupes de Lie résolubles. Bull. Soc. Math. France 85 (1957), 113-121.

[5] B. Farb and L. Mosher. On the asymptotic geometry of abelian-by-cyclic groups. Acta Math. 184(2) (2000), 145-202.

[6] F. P. Greenleaf Invariant Means on Topological Groups and Their Applications (Van Nostrand Mathematical Studies, 16). Van Nostrand Reinhold Co., New York, 1969.

[7] S. Helgason. Differential Geometry, Lie Groups and Symmetric Spaces (Graduate Studies in Mathematics, 34). American Mathematical Society, Providence, RI, 2001. Corrected reprint of the 1978 original.

[8] G. Hochschild. The automorphism group of a Lie group. Trans. Amer. Math. Soc. 72 (1952), 209-216.

[9] N. Jacobson. A note on automorphisms and derivations of Lie algebras. Proc. Amer. Math. Soc. 6 (1955), 281-283.

[10] M. Kanai. Rigidity of the Weyl chamber flow, and vanishing theorems of Matsushima and Weil. Ergod. Th. \& Dynam. Sys. 29(4) (2009), 1273-1288.

[11] A. Katok and R. J. Spatzier. First cohomology of Anosov actions of higher rank abelian groups and applications to rigidity. Publ. Math. Inst. Hautes Études Sci. 79 (1994), 131-156.

[12] A. Katok and R. J. Spatzier Subelliptic estimates of polynomial differential operators and applications to rigidity of abelian actions. Math. Res. Lett. 1(2) (1994), 193-202.

[13] B. Kleiner and B. Leeb. Rigidity of quasi-isometries for symmetric spaces and Euclidean buildings. Publ. Math. Inst. Hautes Études Sci. 86 (1997), 115-197 (1998).

[14] A. W. Knapp. Lie Groups Beyond an Introduction (Progress in Mathematics, 140), 2nd edn. Birkhäuser Boston, Inc., Boston, MA, 2002.

[15] A. Kononenko. Infinitesimal rigidity of boundary lattice actions. Ergod. Th. \& Dynam. Sys. 19(1) (1999), 35-60.

[16] H. Maruhashi. Parameter rigid actions of simply connected nilpotent Lie groups. Ergod. Th. \& Dynam. Sys. 33(6) (2013), 1864-1875.

[17] H. Maruhashi. Vanishing of cohomology and parameter rigidity of actions of solvable Lie groups. Geom. Topol. 21(1) (2017), 157-191.

[18] P. Pansu. Métriques de Carnot-Carathéodory et quasiisométries des espaces symétriques de rang un. Ann. Math. (2) 129(1) (1989), 1-60.

[19] A. Reiter Ahlin. The large scale geometry of nilpotent-by-cyclic groups. Preprint, 2005, arXiv:math/ 0507301.

[20] M. Saito. Sur certains groupes de Lie résolubles. II. Sci. Papers Coll. Gen. Ed. Univ. Tokyo 7 (1957), $157-168$.

[21] A. N. Starkov. Rigidity problem for lattices in solvable Lie groups. Proc. Indian Acad. Sci. Math. Sci. 104(3) (1994), 495-514. 